\title{
ON THE RELATIONSHIP BETWEEN DERIVATIONS AND MEASURABLE DIFFERENTIABLE STRUCTURES
}

\author{
Andrea Schioppa \\ New York University, Courant Institute, Department of Mathematics \\ 251 Mercer St., New York, NY 10012, U.S.A.; schioppa@cims.nyu.edu
}

\begin{abstract}
We study the relationship between measurable differentiable structures on doubling metric measure spaces and derivations. We prove:

(1) the existence of a measurable differentiable structure assuming that one can control the pointwise upper Lipschitz constant of a function through derivations;

(2) an extension of a result of Keith about the choice of chart functions.
\end{abstract}

\section{Introduction}

The extension of first order calculus to metric measure spaces which are not smooth has been a topic of research in the last decade. The search for regularity conditions on a metric measure space allowing to generalize results and concepts of first order calculus, for example the notions of derivative and gradient, has been a topic of intensive research. We refer the reader to the survey [Hei07] for more details. A fundamental result about the geometry of Lipschitz functions on Euclidean spaces is the Rademacher Differentiation Theorem which asserts that a Lipschitz function is differentiable a.e. with respect to the Lebesgue measure.

The Rademacher Differentiation Theorem for metric measure spaces. In [Che99] Cheeger formulated an extension of this result to doubling metric measure spaces which admit a weak version of the Poincaré inequality in the sense presented by Heinonen and Koskela in [HK98, Hei01]. The starting point of this generalization is the introduction of a notion of linear independence of Lipschitz functions at a point (compare Definition 4.5). Because of the the Poincare inequality it is possible to prove that there is a uniform bound on the number of Lipschitz functions that are linearly independent on a set of positive measure. Such kind of finite dimensionality result can be interpreted as a Rademacher Differentiation Theorem and used to introduce the notion of a measurable differentiable structure which allows to take partial derivatives with respect to chart functions (see Section 4). In [Kei04a] Keith found a weaker condition, the "Lip-lip" inequality, which implies the existence of a measurable differentiable structure. This condition can be interpreted as a constraint on the oscillation of a Lipschitz function at small scales. The oscillation is of course dependent on the scale, but the "Lip-lip" inequality prevents a Lipschitz function from oscillating a lot on some scales and very little on others. For another account of this result we refer the reader to [KM11]. Keith showed also in [Kei04b] that chart functions can be chosen among distance functions from points. His argument assumed a Poincaré inequality and exploited Sobolev spaces techniques.

doi:10.5186/aasfm.2014.3910

2010 Mathematics Subject Classification: Primary 53C23, 46J15. algebra.

Key words: Measurable differentiable structure, Rademacher Theorem, derivation, Lipschitz 
Derivations. One can introduce a notion of "derivatives" on metric measure spaces without requiring much regularity on the metric space, but the construction can then become trivial. In [Wea00] Weaver introduced a concept of derivation which can be thought as an extension of the concept of a measurable vector field on a Lipschitz manifold to a metric measure space. Cheeger and Weaver proved that their constructions agree for the spaces considered in [Che99]: details can be found in [Wea00, sec. 5, example F]. However, the relationship between measurable differentiable structures and derivations is still unclear. We were motivated to study this relation by the work of Gong [Gon11] which produces bounds on the number of independent derivations on a doubling metric measure space and recovers a finite dimensionality result from a "Lip-derivation" inequality.

Main results. We summarize here the results of this work and refer the reader to the corresponding sections for explanations of the terminology. It is first necessary to investigate the linear algebraic structure of the derivation module. In [Wea00, Theorem 10] it is shown that one can decompose a finitely generated abelian $W^{*}$ module over $L^{\infty}(X)$ into the module of measurable sections of some bundle of finite dimensional Banach spaces. The point of view here is slightly different because we are not interested in choosing a norm or an explicit realization of the bundle as in [Wea00]. We are interested in a decomposition into free modules and in developping some linear algebra tools needed in the following sections, in particular Corollary 2.26 corresponding to the "Gram-Schmidt" orthogonalization for derivations in [Gon11].

Theorem 2.42. Suppose that the module of derivations $\operatorname{Der}(X, \mu)$ has index locally bounded by $N$. Then there is a measurable partition

$$
X=X_{0} \sqcup \cdots \sqcup X_{N} \sqcup \Omega,
$$

such that

- $\mu(\Omega)=0$;

- if $X_{i} \neq \emptyset$, the $\mathrm{L}^{\infty}\left(X_{i}, \mu\right)$ module $\operatorname{Der}\left(X_{i}, \mu\right)$ is free of rank $i$.

A basis for $\operatorname{Der}\left(X_{i}, \mu\right)$ will be called a local basis of derivations.

We then show the existence of a measurable differentiable structure assuming that derivations control the pointwise upper Lipschitz constant $£ f$ (defined in Sec. 3) of the function. There are different motivations for this result. The Lip-lip inequality can be regarded as an infinitesimal version of the Poincaré inequality sufficient for the existence of a differentiable structure. One might then wonder if there is a similar condition involving derivations. For instance, in [Wea00, Definition 30] the concept of rigidly differentiable space is introduced. In this case derivations control the global Lipschitz constant of the functions and the same argument used to show that a Poincaré inequality space is quasiconvex implies that a rigidly differentiable complete metric space is a length space. In the case of metric differentiable structures one might expect to weaken the condition to allow a local bound on the pointwise upper Lipschitz constant. Moreover, it is no longer necessary to assume a uniform bound, but the conformal factor $\lambda$ can vary with the point (but nevertheless the bound on the dimension of the differentiable structure does not depend on $\lambda$; in the case of the "Lip-lip" inequality the bound produced by Keith depends on the "Lip-lip" constant):

Theorem 5.9. Let $(X, \rho, \mu)$ be a doubling metric measure space. Assume that: 
- there are $N$ derivations $D_{1}, \cdots, D_{n}$ and a nowhere vanishing $\lambda \in \mathrm{L}^{\infty}(X, \mu)$;

- for any Lipschitz function $f$, there is a set $\Omega_{f}$ such that

$$
\begin{aligned}
\mu\left(\Omega_{f}\right) & =0 ; \\
\max _{j=1, \cdots, N}\left|D_{j} f(x)\right| & \geq \lambda(x) £ f(x) \quad \forall x \in X \backslash \Omega_{f} ;
\end{aligned}
$$

then $X$ admits of a measurable differentiable structure whose dimension is at most $N$.

The last result is an extension of the result of [Kei04b] about the choice of the chart functions.

Theorem 6.26. Suppose the doubling metric measure space $(X, \rho, \mu)$ admits a measurable differentiable structure and that for each chart $\left(X_{\alpha},\left\{x_{\alpha}^{j}\right\}_{j=1}^{N_{\alpha}}\right)$ the partial derivatives are derivations. If $\left\{g_{j}\right\}_{j=1}^{M}$ is a generating set for the Lipschitz algebra $\operatorname{Lip}^{\infty}(X)$, the charts can be chosen so that the chart functions belong to $\left\{g_{j}\right\}_{j=1}^{M}$.

A simple example of a metric measure space to which this result applies is a totally disconnected subset $K$ of $\mathbf{R}^{n}$ with positive Lebesgue measure. Denoting by $\rho$ the Euclidean distance and by $\mu$ the Lebesgue measure, $(K, \rho, \mu)$ admits a differentiable structure by the classical Rademacher Theorem. Note that $K$ does not admit a Poincaré inequality, for example observing that a minimal upper gradient for $\chi_{K}$ is identically zero. Given $f \in \operatorname{Lip}^{\infty}(K), \frac{\partial f}{\partial x^{i}} \in \mathrm{L}^{\infty}(K, \mu)$ and it is easy to see that partial derivatives with respect to the coordinate functions $\frac{\partial}{\partial x^{i}}$ are derivations. Another family of examples is obtained by considering $K$ totally disconnected in a Carnot group and using horizontal vector fields to define derivations; compare [Wea00, Sec. 5D] for a discussion of derivations in subRiemannian geometry.

Organization of the paper. In Section 2 we discuss the linear algebraic structure of the module of derivations. The module of derivations is a module over $L^{\infty}(X)$. The ring $L^{\infty}(X)$ is not an integral domain and some care must be taken in introducing notions like "basis" or "rank". We decided to restrict the term "basis" to the case in which the module is free and replace "rank" by index (so the terminology is different from that used in [Gon11]).

In Section 3 we recall some results about the local Lipschitz constants of a function. We then prove, assuming that the metric space is doubling, the localized derivation inequality (3.18) which, roughly speaking, says that if we apply a derivation $D$ to a Lipschitz function $f$, the size of $D f$ is locally controlled by the local Lipschitz constant $£ f$.

In Section 4 we recall background material about measurable differentiable structures. In particular, to a metric measure space with a measurable differentiable structure it is possible to associate a measurable cotangent bundle and use this to construct (reflexive) Sobolev spaces $\mathrm{H}^{1, p}$ for $p>1$. We choose a different class of Sobolev spaces from that employed by Cheeger because the minimal upper gradient might become trivial if the space lacks enough rectifiable curves. An example to keep in mind is a positive Lebesgue measure Cantor set in $[0,1]$ : it has a measurable differentiable structure as it is a positive Lebesgue measure subset of $[0,1]$ but the corresponding $\mathrm{H}^{1, p}$ does not inject into the corresponding $\mathrm{L}^{p}$ space. The question of injectivity is closely related to the closability of the exterior differential $d$ coming from the measurable differentiable structure (Proposition 4.26). 
In Section 5 we present a finite dimensionality result, i.e. the existence of a measurable differentiable structure, assuming that the metric measure space is doubling and the "reverse infinitesimal derivation inequality" (5.11) holds. This condition, roughly speaking, says that there are sufficiently many derivations to control the size of the local Lipschitz constant $£ f$ up to an $\mathrm{L}^{\infty}$ conformal factor $\lambda$ (uniform in the sense that does not depend on the Lipschitz function). The reverse infinitesimal derivation inequality should be compared with the "Lip-derivation" inequality of [Gon11]. Our argument differs from that used by Gong to prove finite dimensionality as we do not use an embedding into Euclidean space but we exploit the linear algebraic structure of the derivation module and the localized derivation inequality.

In Section 6 we extend the results of Keith [Kei04b] about the choice of the chart functions. We first present a representation formula (6.3) of derivations in terms of partial derivatives. We then show that if the partial derivatives are derivations the existence of a measurable differentiable structure is equivalent to the reverse infinitesimal derivation inequality. We give sufficient conditions for partial derivatives to be derivations but we are not able to settle the question as Sobolev space techniques seem insufficient if $\mathrm{H}^{1, p}$ does not inject in $\mathrm{L}^{p}$. We then generalize the result of Keith on the choice of chart functions (Theorem 6.26) using Lipschitz algebra techniques.

\section{Derivations and linear algebra}

In this section we first recall the definition and some properties of the Lipschitz algebra $\operatorname{Lip}^{\infty}(X)$ of a metric space $(X, \rho)$. We then recall the definition of the $\mathrm{L}^{\infty}(X, \mu)$-module $\operatorname{Der}(X, \mu)$ of derivations of a metric measure space $(X, \rho, \mu)$. Derivations form a module over the ring of essentially bounded functions. We proceed to investigate the algebraic structure of this module using linear algebra and measure theory. In particular, we give conditions to decompose the module of derivations into free modules over "smaller rings" $\mathrm{L}^{\infty}(U, \mu)$ where $U \subset X$ has positive measure (Theorem 2.42). An example to keep in mind is that of smooth vector fields defined on a smooth manifold $M$. In that case one replaces $\operatorname{Lip}^{\infty}(X)$ by the algebra of bounded smooth functions and $\operatorname{Der}(X, \mu)$ by the $C^{\infty}(M)$-module of smooth vector fields.

Definition 2.1. (Lipschitz algebra) Let $(X, \rho)$ be a metric space. We denote the collection of bounded real-valued Lipschitz functions on $(X, \rho)$ by $\operatorname{Lip}^{\infty}(X)$, which is a real algebra.

Definition 2.2. For a Lipschitz function $f: X \rightarrow \mathbf{R}$ we denote by $\mathbf{L}(f)$ its global Lipschitz constant:

$$
\mathbf{L}(f)=\sup _{x \neq y} \frac{|f(x)-f(y)|}{\rho(x, y)} .
$$

For $f \in \operatorname{Lip}^{\infty}(X)$ we define the norm

$$
\|f\|_{\operatorname{Lip}^{\infty}(X)}=\|f\|_{\infty} \vee \mathbf{L}(f) .
$$

This gives $\left(\operatorname{Lip}^{\infty}(X),\|\cdot\|_{\operatorname{Lip}^{\infty}(X)}\right)$ the structure of a Banach algebra, compare [Wea99, Sec. 4.1].

As Weaver points out in [Wea99, Sec. 4.1], the term "Banach algebra" is used slightly differently in this context as $\operatorname{Lip}^{\infty}(X)$ is actually bi-Lipschitz to a Banach algebra in the usual sense. An important property of $\operatorname{Lip}^{\infty}(X)$ is that it is a dual Banach space and it has a unique predual. The are two approaches to prove this 
result. The first approach uses the de Leeuw's map [dL62]. The second approach gives an explicit description of the dual space in terms of the Arens-Eells space [AE56]. For more information we refer the reader to [Wea99, Chap. 2]. As $\operatorname{Lip}^{\infty}(X)$ is a dual Banach space with a unique predual, we can consider the weak* topology on it. It turns out that $f_{n} \rightarrow f$ in the weak* topology if and only if $f_{n} \rightarrow f$ pointwise and if $\sup _{n} \mathbf{L}\left(f_{n}\right)<\infty$.

Definition 2.4. A set $\left\{g_{j}\right\}_{j=1}^{M} \subset \operatorname{Lip}^{\infty}(X)$ is called a generating set for $\operatorname{Lip}^{\infty}(X)$ if the subalgebra generated by it is weak* dense in $\operatorname{Lip}^{\infty}(X)$.

An important result connected to the previous definition is the Stone-Weierstraß Theorem 6.30. Following Weaver we define derivations: [Wea00].

Definition 2.5. (Derivations) Let $(X, \rho, \mu)$ be a separable metric measure space with $\mu$ Borel and $\sigma$-finite. A map

$$
D: \operatorname{Lip}^{\infty}(X) \rightarrow \mathrm{L}^{\infty}(X, \mu),
$$

is called a derivation if:

- it is linear and bounded;

- it satisfies the product rule

$$
D(f g)(x)=D f(x) g(x)+f(x) D g(x)
$$

- if $f_{n} \rightarrow f$ in the weak* topology in $\operatorname{Lip}^{\infty}(X)$, then $D f_{n} \rightarrow D f$ in the weak* topology in $\mathrm{L}^{\infty}(X, \mu)$.

The set of all derivations is denoted by $\operatorname{Der}(X, \mu)$ and is an $\mathrm{L}^{\infty}(X, \mu)$-module. If we restrict the Lipschitz functions to a measurable subset of $U \subset X$ we denote the $\mathrm{L}^{\infty}(U, \mu)$-module of derivations by $\operatorname{Der}(U, \mu)$.

In the subsequent sections we will often assume, to simplify the notation in the proofs, that the metric spaces considered are bounded and the measures are finite, this being possible by taking a countable Borel partition. In particular, note that if $U \subset X$ any $D \in \operatorname{Der}(X, \mu)$ can be restricted to give an element $D \mid U \in \operatorname{Der}(U, \mu)$. If $\tilde{f} \in \operatorname{Lip}^{\infty}(X)$ extends $f \in \operatorname{Lip}^{\infty}(U)$, then one defines

$$
D|U(f)=(D \tilde{f})| U \quad(\text { restricted on } U) .
$$

This definition is well-posed by the locality result Proposition 3.16. An alternative might have been to define derivations as maps

$$
D: \operatorname{Lip}_{\text {loc }}(X) \rightarrow \mathrm{L}_{\text {loc }}^{\infty}(X, \mu),
$$

where $\operatorname{Lip}_{\text {loc }}(X)$ denotes the algebra of locally Lipschitz functions (i.e. $f \in \operatorname{Lip}_{\text {loc }}(X)$ if for each point there is a neighbourhood on which $f$ is Lipschitz), and $\mathrm{L}_{\text {loc }}^{\infty}(X, \mu)$ is the algebra of functions which are locally essentially bounded.

For $U \subset X, \mu(U)>0, \mathrm{~L}^{\infty}(U, \mu)$ is a commutative ring with unity. The subset

$$
\mathcal{V}^{\infty}(U, \mu)=\left\{f \in \mathrm{L}^{\infty}(U, \mu): \mu(\{x \in U: f(x)=0\})=0\right\}
$$

consists of those functions which are non vanishing (essentially). We define the set $\mathcal{V}_{M}^{\infty}(U, \mu)$ of those functions whose absolute value is a.e. bounded from below by $M>0$ :

$$
\mathcal{V}_{M}^{\infty}(U, \mu)=\left\{f \in \mathrm{L}^{\infty}(U, \mu): \mu(\{x \in U:|f(x)|<M\})=0\right\} .
$$

Note that $f \in \mathrm{L}^{\infty}(U, \mu)$ is a unit if and only if $f \in \mathcal{V}_{M}^{\infty}(U, \mu)$ for some $M>0$. 
Lemma 2.12. Let $V_{1}, \cdots, V_{M}: X \rightarrow \mathcal{V}$ be Borel maps, with $(\mathcal{V},\|\cdot\|)$ a normed vector space and $\left\|V_{i}\right\| \in \mathrm{L}^{\infty}(X, \mu)$. Suppose that $\mu(A)>0$ and $\forall x \in A$

$$
\mathbf{R}\left\langle V_{1}(x), \cdots, V_{M}(x)\right\rangle \quad \text { (real span) }
$$

has dimension $M-1$; then there are Borel functions $\lambda_{i} \in \mathrm{L}^{\infty}(A, \mu)$ such that

$$
\left\|\lambda_{i}\right\|_{\mathrm{L}^{\infty}(A, \mu)} \leq 1
$$

and

$$
\begin{aligned}
\sum_{i=1}^{M} \lambda_{i}(x) V_{i}(x) & =0 \quad \text { for a.e. } x \in A, \\
\mu\left(\left\{x: \forall i, \lambda_{i}(x)=0\right\}\right) & =0 .
\end{aligned}
$$

Furthermore, if $\forall x \in A$

$$
\mathbf{R}\left\langle V_{1}(x), \cdots, V_{M}(x)\right\rangle=\mathbf{R}\left\langle V_{1}(x), \cdots, V_{M-1}(x)\right\rangle,
$$

then $\lambda_{M} \in \mathcal{V}^{\infty}(A, \mu)$.

Proof. For almost every point $x \in X$ we have $\left\{\alpha_{1}(x), \cdots \alpha_{M}(x)\right\} \subset \mathbf{R}$ such that

$$
\left(\alpha_{1}(x), \cdots, \alpha_{M}(x)\right) \neq 0,
$$

and

$$
\sum_{i=1}^{M} \alpha_{i}(x) V_{i}(x)=0 .
$$

Note that if the additional hypothesis (2.16) holds, we can assume that

$$
\alpha_{M}(x) \neq 0 .
$$

Once the proof is complete, this will imply that $\lambda_{M}$ is nowhere vanishing.

By assumption any other $M$-tuple such that (2.17) holds is a multilple of

$$
\left(\alpha_{1}(x), \cdots, \alpha_{M}(x)\right) .
$$

Therefore, the map

$$
\begin{aligned}
\Lambda: X & \rightarrow \mathbf{R P}^{M-1} \quad \text { (real projective space) } \\
x & \mapsto\left[\left(\alpha_{1}(x), \cdots, \alpha_{M}(x)\right)\right] ;
\end{aligned}
$$

is well-defined (a.e. as we need the $\left\|V_{i}(x)\right\|$ to be finite). We define

$$
\begin{aligned}
& G: \mathbf{R P}^{M-1} \times X \rightarrow \mathbf{R} \\
& (\sigma, x) \mapsto \max \left\{\left\|\sum_{i=1}^{M} \alpha_{i} V_{i}(x)\right\|:\left(\alpha_{i}\right) \in \mathbf{R}^{M}:\left|\left(\alpha_{i}\right)\right| \leq 1 \text { and }\left[\left(\alpha_{i}\right)\right]=\sigma\right\} ;
\end{aligned}
$$

then $G$ is continuous in $\sigma$ and Borel measurable in $x$. To show that $\Lambda$ is Borel measurable, it suffices to show that $\Lambda^{-1}(C)$ is a Borel set whenever $C \subset \mathbf{R P}^{M-1}$ is closed. If $\left\{\alpha_{i}\right\} \subset C$ be a countable dense subset, then

$$
\Lambda^{-1}(C)=\bigcap_{n} \bigcup_{i}\left\{x: G\left(\alpha_{i}, x\right)<\frac{1}{n}\right\} .
$$


Therefore $\Lambda$ is a Borel function. Finally, $\mathbf{R P}^{M-1}$ can be covered by $M$ differentiable charts. On each chart $\Lambda$ can be lifted to $\left(\lambda_{1}, \cdots, \lambda_{M}\right)$ with

$$
\left\|\lambda_{i}\right\|_{L^{\infty}(X, \mu)} \leq 1
$$

Definition 2.23. The derivations $\left\{D_{1}, \cdots, D_{n}\right\} \subset \operatorname{Der}(U, \mu)$ are said to be linearly independent (over $\mathrm{L}^{\infty}(U, \mu)$ ) if for any $\left\{\lambda_{1}, \cdots, \lambda_{n}\right\} \subset \mathrm{L}^{\infty}(U, \mu)$,

$$
\sum_{i=1}^{n} \lambda_{i} D_{i}=0,
$$

implies that $\lambda_{i}=0$. This means that for any choice of the representatives for the $\lambda_{i}$, these vanish a.e. In the sequel, we have not kept the distinction between elements of $\mathrm{L}^{p}$-spaces and their representatives.

Definition 2.25. (Finite index) If in $\operatorname{Der}(U, \mu)$ any linearly independent set of derivations has at most $N$ elements, $\operatorname{Der}(U, \mu)$ is said to have finite index. The smallest value of $N$ is the index of $\operatorname{Der}(U, \mu)$.

The previous definition of index is an attempt to generalize the notion of the rank of a free module. If $\operatorname{Der}(U, \mu)$ were free, i.e. if $\operatorname{Der}(U, \mu)$ had a basis over $\mathrm{L}^{\infty}(U, \mu)$, rank and index would agree. We now prove an implication of the condition that $\operatorname{Der}(U, \mu)$ has finite index. We first assume that $\operatorname{Lip}^{\infty}(X)$ has a finite generating set $\left\{g_{j}\right\}_{j=1}^{M}(M<\infty)$. We will then reformulate this result for the case in which $\left\{g_{j}\right\}_{j=1}^{M}$ is countable.

Proposition 2.26. Suppose that $\left\{D_{i}\right\}_{i=1}^{n} \subset \operatorname{Der}(U, \mu)$ is a linearly independent set. Suppose $\operatorname{Lip}^{\infty}(U)$ has a finite generating set $\left\{g_{j}\right\}_{j=1}^{M}$. Then for a.e. $x \in U$ the row vectors

$$
D_{i} g(x) \equiv\left(D_{i} g_{1}(x), \cdots, D_{i} g_{M}(x)\right)
$$

are linearly independent. In particular, $M \geq n$.

Proof. Let $V_{i}=D_{i} g$. Suppose that there is a measurable $V \subset U$ such that $\mu(V)>0$ and $\forall x \in V$

$$
\mathbf{R}\left\langle V_{1}(x), \cdots, V_{n}(x)\right\rangle
$$

has dimension strictly smaller than $n$. Without loss of generality we can assume that $\forall x \in V$

$$
\mathbf{R}\left\langle V_{1}(x), \cdots, V_{k}(x)\right\rangle
$$

has dimension $k-1$. We now apply Lemma 2.12 with $\mathcal{V}=\mathbf{R}^{M}$ obtaining

$$
\left\{\lambda_{1}, \cdots, \lambda_{k}\right\} \subset \mathrm{L}^{\infty}(V, \mu)
$$

with

$$
\begin{aligned}
\sum_{i=1}^{k} \lambda_{i}(x) V_{i}(x) & =0 \\
\mu\left(\left\{x: \forall i, \lambda_{i}(x)=0\right\}\right) & =0 .
\end{aligned}
$$


We extend the $\lambda_{i}$ to $\mathrm{L}^{\infty}(U, \mu)$ by setting them equal to 0 on $U \backslash V$. The conclusion is that the derivation

$$
D^{\prime}=\sum_{i=1}^{k} \lambda_{i} D_{i}
$$

maps each $g_{i}$ to 0 . By the product rule, $D^{\prime}=0$ on the algebra generated by $\left\{g_{j}\right\}_{j=1}^{M}$. But by weak* continuity, the derivation $D^{\prime}$ is trivial on $\operatorname{Lip}^{\infty}(U)$. Therefore the derivations $D_{1}, \cdots, D_{k}$ are linearly depedent, contrary to the hypothesis. Note also that as the $V_{i}$ have to be linearly independent a.e., $M \geq n$.

Corollary 2.30. Suppose that $\left\{D_{i}\right\}_{i=1}^{n} \subset \operatorname{Der}(U, \mu)$ is a linear independent set, $U$ Borel with $\mu(U)>0$. Let $\left\{g_{j}\right\}_{j=1}^{M}$ be a finite generating set for $\operatorname{Lip}^{\infty}(U)$. Then there are:

- a subset of the generators $\left\{g_{1}^{\prime}, \cdots, g_{n}^{\prime}\right\} \subset\left\{g_{j}\right\}_{j=1}^{M}$;

- a Borel measurable $V \subset U$ with $\mu(V)>0$;

- an invertible $n \times n$ matrix $A$ over the ring $\mathrm{L}^{\infty}(V, \mu)$, that is,

$$
A=\left(a_{i j}\right)_{i, j=1, \cdots, n}
$$

for $a_{i j} \in \mathrm{L}^{\infty}(V, \mu)$ such that if we define a new set of derivations $\left\{D_{i}^{\prime}\right\}_{i=1}^{n} \subset$ $\operatorname{Der}(U, \mu)$ by

$$
A D \equiv D^{\prime}
$$

then $\forall x \in V$

$$
D_{i}^{\prime} g_{j}^{\prime}(x)=\delta_{i, j}
$$

Proof. By Proposition 2.26 for a.e. $x \in U$ the $D_{i} g(x)$ are linearly independent. Therefore there are

- a measurable $U^{\prime} \subset U$ with $\mu\left(U^{\prime}\right)>0$ and

- a subset $\left\{g_{1}^{\prime}, \cdots, g_{n}^{\prime}\right\} \subset\left\{g_{j}\right\}_{j=1}^{M}$,

such that the matrix

$$
B(x)=\left(\begin{array}{ccc}
D_{1} g_{1}^{\prime}(x) & \cdots & D_{1} g_{n}^{\prime}(x) \\
\vdots & \vdots & \vdots \\
D_{n} g_{1}^{\prime}(x) & \cdots & D_{n} g_{n}^{\prime}(x)
\end{array}\right)
$$

is non-singular for each $x \in U^{\prime}$. In particular, $\operatorname{det} B \neq 0$ on $U^{\prime}$ so we can find $\varepsilon>0$ and $V \subset U^{\prime}$ with $\mu(V)>0$ and $|\operatorname{det} B|>\varepsilon$ on $V$. As $\operatorname{det} B \in \mathcal{V}_{\varepsilon}^{\infty}(V, \mu)$, we have that

$$
f(x)=\frac{1}{\operatorname{det} B(x)} \in \mathrm{L}^{\infty}(V, \mu)
$$

If we let $C$ be the cofactor matrix of $B$ and define new derivations in $\operatorname{Der}(V, \mu)$ by

$$
D_{i}^{\prime}=\left.\sum_{j=1}^{n} f C_{i j} D_{j}\right|_{V}
$$

we have that (2.33) holds a.e. in $V$. We finally let $A=f C$.

We now discuss the modifications for the case $M=\infty$ by which we mean that $\left\{g_{j}\right\}_{j=1}^{M}$ is countable. 
Proposition 2.35. Suppose that $\left\{D_{i}\right\}_{i=1}^{n} \subset \operatorname{Der}(U, \mu)$ is a linearly independent set, $U$ Borel. Let $\left\{g_{j}\right\}_{j=1}^{\infty}$ be a countable generating set for $\operatorname{Lip}^{\infty}(U)$ with

$$
\sup _{j=1, \cdots, \infty}\left\|g_{j}\right\|_{\operatorname{Lip}^{\infty}(X)} \leq C<\infty .
$$

Then for a.e. $x \in U$ the row vectors

$$
D_{i} g(x) \equiv\left(D_{i} g_{j}(x)\right)_{j=1}^{\infty} \in l^{\infty}(\mathbf{N})
$$

are linearly independent. Furthermore there are $\left\{g_{1}^{\prime}, \cdots, g_{n}^{\prime}\right\}, V$ and $A$ such that the conclusions of Corollary 2.30 hold.

Proof. The proof of the first part is like that of Proposition 2.26 but we take $\mathcal{V}=l^{\infty}(\mathbf{N})$. For the proof of the second part we could argue as in Corollary 2.30 provided that there are a subset of the generators $\left\{g_{1}^{\prime}, \cdots, g_{n}^{\prime}\right\}$ and a set $U^{\prime}$ of positive measure such that

$$
B(x)=\left(\begin{array}{ccc}
D_{1} g_{1}^{\prime}(x) & \cdots & D_{1} g_{n}^{\prime}(x) \\
\vdots & \vdots & \vdots \\
D_{n} g_{1}^{\prime}(x) & \cdots & D_{n} g_{n}^{\prime}(x)
\end{array}\right)
$$

is nonsingular on $U^{\prime}$. We prove this arguing by contradiction. Let $T_{M}: l^{\infty}(\mathbf{N}) \rightarrow$ $l^{\infty}(\mathbf{N})$ be the truncation map:

$$
T_{M}\left(c_{i}\right)_{i=1}^{\infty}=\left(c_{1}, c_{2}, \cdots, c_{M}, 0,0, \cdots\right) .
$$

If we cannot find a subset $U^{\prime} \subset U$ and a subset of the generators such that $B$ is nonsingular on $U^{\prime}$, then for each $M$ the vectors $T_{M}\left(D_{i} g\right)$ are linearly dependent a.e. This implies that there is a subset $\tilde{U} \subset U$ with $\mu(U \backslash \tilde{U})=0$ and for each $M \geq 1$ and $x \in \tilde{U}$, the vector subspace

$$
\Lambda_{M}(x)=\left\{\left(\lambda_{1}, \cdots, \lambda_{n}\right) \in \mathbf{R}^{n}: \sum_{i=1}^{n} \lambda_{i} T_{M}\left(D_{i} g\right)(x)=0\right\}
$$

has dimension at least 1 . Now, $\Lambda_{M}(x) \supset \Lambda_{M+1}(x)$ and so

$$
\bigcap_{M=1}^{\infty} \Lambda_{M}(x) \neq\{0\}
$$

as the dimension of a vector subspace of $\mathbf{R}^{n}$ has to lie in $\{0,1, \cdots, n\}$ and the dimension of $\bigcap_{M=1}^{k} \Lambda_{M}(x)$ can decrease only by integer values as we increase $k$. But then (2.40) would imply that the $D_{i} g(x)$ are linearly dependent.

Note that in Proposition 2.26 the role of generators and derivations is not symmetrical. For example, we can have $M>n$ (trivially if $M=\infty$ as in Proposition 2.35). An example is the standard Cantor set whose Lipschitz algebra is generated by the single function $x$ and where all derivations are trivial (see [Wea00, Section 5. Examples A] or use the localized derivation inequality (3.18) which is proved in the next section). Note that the bound $M>n$ for $M$ finite implies the hypothesis of Theorem 2.42 .

Definition 2.41. (Modules of derivations whose index is locally bounded) We say that the module of derivations $\operatorname{Der}(X, \mu)$ has index locally bounded by $N$ if for any set $U \subset X$ of positive measure, $\operatorname{Der}(U, \mu)$ has index at most $N$ over $\mathrm{L}^{\infty}(U, \mu)$. 
Theorem 2.42. Suppose that the module of derivations $\operatorname{Der}(X, \mu)$ has index locally bounded by $N$. Then there is a measurable partition

$$
X=X_{0} \sqcup \cdots \sqcup X_{N} \sqcup \Omega,
$$

such that:

- $\mu(\Omega)=0$;

- if $X_{i} \neq \emptyset$ the $\mathrm{L}^{\infty}\left(X_{i}, \mu\right)$ module $\operatorname{Der}\left(X_{i}, \mu\right)$ is free of rank $i$.

A basis for $\operatorname{Der}\left(X_{i}, \mu\right)$ will be called a local basis of derivations.

Proof. Recall that we assume $\mu(X)<\infty$. Let $\mathcal{R}_{N}(X)$ denote the collection of subsets $U \subset X$ satisfying the following properties:

- $U$ is measurable and $\mu(U)>0$;

- $\operatorname{Der}(U, \mu)$ has index $N$;

if $\mathcal{R}_{N}(X)=\emptyset$ we let $X_{N}=\emptyset$. If $\mathcal{R}_{N}(X) \neq \emptyset$, let $U_{N, 1} \in \mathcal{R}_{N}(X)$ be such that

$$
\mu\left(U_{N, 1}\right)>\frac{2}{3} \sup _{V \in \mathcal{R}_{N}(X)} \mu(V) .
$$

If

$$
\mathcal{R}_{N}\left(X \backslash U_{N, 1}\right)=\emptyset
$$

we stop; otherwise we select $U_{N, 2} \in \mathcal{R}_{N}\left(X \backslash U_{N, 1}\right)$ with

$$
\mu\left(U_{N, 2}\right)>\frac{2}{3} \sup _{V \in \mathcal{R}_{N}\left(X \backslash U_{N, 1}\right)} \mu(V) .
$$

The construction of the sets $\left\{U_{N, i}\right\}$ proceeds by induction. There are two cases: either we stop after $N^{\prime}$ steps or we continue up to infinity. In the first case we let

$$
X_{N}=U_{N, 1} \sqcup \cdots \sqcup U_{N, N^{\prime}}
$$

and observe that

$$
\mathcal{R}_{N}\left(X \backslash X_{N}\right)=\emptyset \text {. }
$$

In the second case, as the sets $\left\{U_{N, i}\right\}$ are disjoint and as $\mu(X)<\infty$, we conclude that

$$
\lim _{i \rightarrow \infty} \mu\left(U_{N, i}\right)=0
$$

We now observe that

$$
\begin{aligned}
\sup _{V \in \mathcal{R}_{N}\left(X \backslash \bigsqcup_{i=1}^{\infty} U_{N, i}\right)} \mu(V) & \leq \sup _{V \in \mathcal{R}_{N}\left(X \backslash \bigsqcup_{i=1}^{k} U_{N, i}\right)} \mu(V) \\
& \leq \frac{3}{2} \mu\left(U_{N, k+1}\right) \rightarrow 0
\end{aligned}
$$

as $k \nearrow \infty$; this shows that

$$
\mathcal{R}_{N}\left(X \backslash \cup_{i} U_{N, i}\right)=\emptyset .
$$

The conclusion is that if we let $X_{N}=\sqcup_{i=1}^{\infty} U_{N, i}$ then there is no measurable subset $V \subset X \backslash X_{N}$ such that $\mu(V)>0$ and $\operatorname{Der}(V, \mu)$ has index $N$. We now show that $\operatorname{Der}\left(X_{N}, \mu\right)$ has index $N$. We first show that the index is at most $N$. Given a linearly independent set $\left\{D_{1}, \cdots, D_{m}\right\} \subset \operatorname{Der}\left(X_{N}, \mu\right)$ we can consider the restrictions 
$\chi_{U_{N, 1}} D_{i} \in \operatorname{Der}\left(U_{N, 1}, \mu\right)$. We claim that these are linearly independent in $\operatorname{Der}\left(U_{N, 1}, \mu\right)$; otherwise there would be $\lambda_{i} \in \mathrm{L}^{\infty}\left(U_{N, 1}, \mu\right)$ with

$$
\sum_{i=1}^{m} \lambda_{i} \chi_{U_{N, 1}} D_{i}=0
$$

with some $\lambda_{i_{0}} \neq 0$. But extending each $\lambda_{i}$ to an element of $\mathrm{L}^{\infty}\left(X_{N}, \mu\right)$, namely, by letting it be 0 on the complement of $U_{N, 1}$, we would obtain a linear dependence relation

$$
\sum_{i=1}^{m} \lambda_{i} D_{i}=0
$$

in $\operatorname{Der}\left(X_{N}, \mu\right)$. This would contradict the linear independence of the $D_{i}$. Finally, as the index of $\operatorname{Der}\left(U_{N, 1}, \mu\right)$ is $N, m \leq N$. We now show that there are $N$ linearly independent derivations in $\operatorname{Der}\left(X_{N}, \mu\right)$. For each $j$ choose $N$ independent derivations $\left\{D_{1, j}, \cdots, D_{N, j}\right\} \subset \operatorname{Der}\left(U_{N, j}, \mu\right)$. Without loss of generality we can assume that $\left\|D_{i, j}\right\| \leq 1$. As each function in $\mathrm{L}^{\infty}\left(U_{N, j}, \mu\right)$ can be extended to $\mathrm{L}^{\infty}\left(X_{N}, \mu\right)$ by setting it equal to 0 on the complement of $U_{N, j}$, we get extensions $\chi_{U_{N, j}} D_{i, j} \in \operatorname{Der}\left(X_{N}, \mu\right)$ (with slight abuse of notation). Therefore

$$
D_{i}=\sum_{j} \chi_{U_{N, j}} D_{i, j}
$$

defines an element of $\operatorname{Der}\left(X_{N}, \mu\right)$ with norm at most 1 . The $D_{i}$ are independent: assuming that there are $\lambda_{i} \in \mathrm{L}^{\infty}\left(X_{N}, \mu\right)$ such that $\lambda_{i_{0}} \neq 0$ and

$$
\sum_{i} \lambda_{i} D_{i}=0
$$

for some index $j, \chi_{U_{N, j}} \lambda_{i_{0}} \neq 0$ in $\mathrm{L}^{\infty}\left(U_{N, j}, \mu\right)$. But we would then obtain a dependence relation

$$
\sum_{i} \chi_{U_{N, j}} \lambda_{i} D_{i, j}=0
$$

in $\operatorname{Der}\left(U_{N, j}, \mu\right)$. The sets $X_{N-1}, X_{N-2}, \cdots, X_{0}$ are constructed by induction. Here we use the hypothesis that the index is locally bounded by $N$. For example, this implies that $X \backslash X_{N}$ has index locally bounded by $N-1$. The induction step proceeds as follows. Suppose we have already constructed $X_{N}, \cdots, X_{N-k}$ and $N-k>0$. If we let

$$
Y=X \backslash\left(X_{N-k} \sqcup \cdots \sqcup X_{N}\right),
$$

then $Y$ has index locally bounded by $N-k-1$. Let $\mathcal{R}_{N-k-1}(Y)$ denote the collection of subsets $U \subset Y$ satisfying the following properties:

- $U$ is measurable and $\mu(U)>0$;

- $\operatorname{Der}(U, \mu)$ has index $N-k-1$;

then we apply the same argument used to construct $X_{N}$. In particular, there is no measurable subset $V \subset Y \backslash X_{N-k-1}$ such that $\mu(V)>0$ and $\operatorname{Der}(V, \mu)$ has index $N-k-1$. Before proceeding further, we remark that $X_{0}$ might be nonempty. We now show that

$$
\mu\left(X \backslash\left(X_{0} \sqcup \cdots \sqcup X_{N}\right)\right)=0
$$


arguing by contradiction. If

$$
\mu\left(X \backslash\left(X_{0} \sqcup \cdots \sqcup X_{N}\right)\right)>0,
$$

there would be some measurable

$$
V \subset X \backslash X_{0} \sqcup \cdots \sqcup X_{N}
$$

with $\mu(V)>0$ and $\operatorname{Der}(V, \mu)$ having index in $\{0, \cdots, N\}$. This contradicts the construction of the $X_{i}$ 's. Note that in this step we have again used that the index is locally bounded by $N$.

We now prove that $\operatorname{Der}\left(X_{k}, \mu\right)$ is free over $\mathrm{L}^{\infty}\left(X_{k}, \mu\right)$. We choose a maximal linearly independent set

$$
\left\{D_{1}, \cdots, D_{k}\right\} \subset \operatorname{Der}\left(X_{k}, \mu\right)
$$

and we show it is a basis. As the elements of this set are linearly independent, it suffices to show that it spans $\operatorname{Der}\left(X_{k}, \mu\right)$ over $\mathrm{L}^{\infty}\left(X_{k}, \mu\right)$. Let $D^{\prime} \in \operatorname{Der}\left(X_{k}, \mu\right)$ and define the collection $\mathcal{S}_{D^{\prime}}\left(X_{k}\right)$ of subsets of $X_{k}$ in the following way: $V \in \mathcal{S}_{D^{\prime}}\left(X_{k}\right)$ if and only if the following holds:

- $V$ is measurable and $\mu(V)>0$;

- there are $\left\{\lambda_{1, V}, \cdots, \lambda_{k, V}\right\} \subset \mathrm{L}^{\infty}\left(X_{k}, \mu\right)$ such that:

$$
\chi_{V} \cdot\left(D^{\prime}-\sum_{j=1}^{k} \lambda_{j, V} D_{j}\right)=0 .
$$

We first show that $\mathcal{S}_{D^{\prime}}\left(X_{k}\right)$ is not empty. By the maximality of $\left\{D_{1}, \cdots, D_{k}\right\}$ there are $\left\{\lambda^{\prime}, \lambda_{1}, \cdots, \lambda_{k}\right\} \subset \mathrm{L}^{\infty}\left(X_{k}, \mu\right)$ such that

$$
\begin{aligned}
& \mu\left(x: \lambda^{\prime}(x)=0\right)=0, \\
& \lambda^{\prime} D^{\prime}-\sum_{j=1}^{k} \lambda_{j} D_{j}=0 .
\end{aligned}
$$

Let $V$ be a measurable set with $\mu(V)>0$ and $\left.\lambda^{\prime}\right|_{V} \in \mathcal{V}_{M}^{\infty}(V, \mu)$ for some $M>0$, implying that $\left.\lambda^{\prime}\right|_{V}$ is invertible in $\mathrm{L}^{\infty}(V, \mu)$. Without loss of generality we can assume that $\left|\lambda^{\prime}(x)\right| \geq M$ for $x \in V$. If we define

$$
\lambda_{j, V}= \begin{cases}\frac{\lambda_{j}(x)}{\lambda^{\prime}(x)} & \text { for } x \in V, \\ 0 & \text { for } x \in{ }^{c} V,\end{cases}
$$

then (2.50) holds implying that $V \in \mathcal{S}_{D^{\prime}}\left(X_{k}\right)$. The same argument used for the set $\mathcal{R}_{N}(X)$ shows that there is a measurable partition

$$
\bigsqcup_{i \in I} U_{i}
$$

of $X_{k}$ such that for each $U_{i}$ there are $\left\{\lambda_{1, U_{i}}, \cdots \lambda_{k, U_{i}}\right\}$ with

$$
\chi_{U_{i}} \cdot\left(D^{\prime}-\sum_{j=1}^{k} \lambda_{j, U_{i}} D_{j}\right)=0 \text {; }
$$

if we let

$$
\lambda_{j}=\sum_{i \in I} \chi_{U_{i}} \lambda_{j, U_{i}}
$$


and sum the equations (2.53) we conclude that

$$
D^{\prime}=\sum_{j=1}^{k} \lambda_{j} D_{j} .
$$

\section{The local Lipschitz constants}

In this section we recall the definition of the local Lipschitz constants $£ f$ and $\ell f$ for a function $f$. Using Egorov and Lusin Theorems, we obtain a measurable decomposition where the local Lipschitz constants behave nicely. In a doubling metric measure space, using the Lebesgue Differentiation Theorem and the locality principle for derivations, we obtain the local estimate (3.18) which we call the localized derivation inequality.

Definition 3.1. [Variation and local Lipschitz constants] Let $f$ be a Lipschitz function. We define the lower and upper variations of $f$ at $x$ from scale $r$ down to 0 by

$$
\begin{aligned}
& £ f(x, r)=\sup _{s \leq r} \sup _{y \in B(x, s)} \frac{|f(x)-f(y)|}{s} \\
& \ell f(x, r)=\inf _{s \leq r} \sup _{y \in B(x, s)} \frac{|f(x)-f(y)|}{s} .
\end{aligned}
$$

Let us define the infinitesimal Lipschitz constants of $f$ at $x$ by

$$
\begin{aligned}
£ f(x) & =\inf _{r \geq 0} £ f(x, r) \\
\ell f(x) & =\sup _{r \geq 0} \ell f(x, r) .
\end{aligned}
$$

As far a we understand, the behaviour of $\ell f$ is not so nice in general. Also, this is not really a local Lipschitz constant. The behaviour of $£ f$ is more regular. For example, if we blow up $f$ near some point, then $£ f$ really is a Lipschitz constant for the blow up. We also note that $£ f$ behaves like a seminorm in $f$ in the following sense, if $f, g$ are Lipschitz functions and $\lambda, \mu \in \mathbf{R}$, then

$$
£(\lambda f+\mu g) \leq|\lambda| £ f+|\mu| £ g .
$$

The proof of the following Lemma is a direct application of Egorov and Lusin Theorems and will be omitted; for details, compare [KM11]:

Lemma 3.7. Let $f$ be a Lipschitz function. Then there is a measurable partition

$$
X=\bigsqcup_{i=1}^{\infty} A_{i} \sqcup \Omega,
$$

such that

- $\Omega$ has measure 0 ,

- $£ f$ and $\ell f$ are continuous on each $A_{i}$,

- $£ f(\cdot, r) \searrow £ f$ and $\ell f(\cdot, r) \nearrow \ell f$ uniformly on each $A_{i}$ for $r \searrow 0$.

The following discussion is not actually needed to prove Theorem 3.18, which is the main result of this section. However, it clarifies the point we made when we said that $£ f(x)$ is essentially the Lipschitz constant of $f$ in a neighbourhood of $x$. 
Definition 3.9. (Local density) Let $(X, \rho)$ be a metric space, $A \subset X$ and $x \in X$. We say that $A$ is locally dense at $x$ if for any $\varepsilon>0$ there is an $r(\varepsilon)>0$ such that if $r \leq r(\varepsilon), A \cap B(x,(1+\varepsilon) r)$ is $\varepsilon r$-dense in $B(x, r)$, in the sense that $\forall y \in B(x, r)$, $\exists z \in A \cap B(x,(1+\varepsilon) r)$ with $\rho(y, z)<\varepsilon r$.

Proposition 3.10. Let $(X, \rho, \mu)$ be a doubling metric measure space and $A \subset X$ a measurable subset. Then for a.e. $x \in A, A$ is locally dense at $x$.

Proof. The case $\mu(A)=0$ is trivial so we assume that $A$ has positive measure. As $\mu$ is doubling, there are constants $C \geq 1$ and $\kappa>0$ such that if $z, w \in X$ and $B(w, s) \subset B(x, r)$, we have

$$
\frac{\mu(B(w, s))}{\mu(B(x, r))} \geq \frac{1}{C}\left(\frac{s}{r}\right)^{\kappa}
$$

let $x \in A$ and suppose that $A \cap B(x,(1+\varepsilon) r)$ is not $\varepsilon r$-dense in $B(x, r)$ In this case, there is a point $y \in B(x, r)$ such that $B(y, \varepsilon r)$ is disjoint from

$$
A \cap B(x,(1+\varepsilon) r) .
$$

As $B(y, \varepsilon r) \subset B(x,(1+\varepsilon) r),(3.11)$ implies that

$$
\frac{\mu(B(x,(1+\varepsilon) r) \backslash A)}{\mu(B(x,(1+\varepsilon) r))} \geq \frac{\mu(B(y, \varepsilon r))}{\mu(B(x,(1+\varepsilon) r))} \geq \frac{1}{C}\left(\frac{\varepsilon}{1+\varepsilon}\right)^{\kappa} ;
$$

as the Lebesgue differentiation theorem holds in the metric measure space $(X, \rho, \mu)$, for a.e. $x \in A, x$ is a density point of $A$, that is,

$$
\lim _{s \searrow 0} \frac{\mu(B(x, s) \backslash A)}{\mu(B(x, s))}=0 .
$$

If we choose $s_{\varepsilon}$ so that $r \leq s_{\varepsilon}$ implies

$$
\frac{\mu(B(x,(1+\varepsilon) r) \backslash A)}{\mu(B(x,(1+\varepsilon) r))}<\frac{1}{C}\left(\frac{\varepsilon}{1+\varepsilon}\right)^{\kappa},
$$

then (3.12) does not hold, impliying that $A \cap B(x,(1+\varepsilon) r)$ is $\varepsilon r$-dense in $B(x, r)$.

Corollary 3.13. Suppose $x_{0} \in A$ is a density point of $A$. Suppose that $f, g$ are Lipschitz function with $f=g$ on $A$. Then

$$
\begin{aligned}
£ f\left(x_{0}\right) & =£ g\left(x_{0}\right), \\
\ell f\left(x_{0}\right) & =\ell g\left(x_{0}\right) .
\end{aligned}
$$

We now come back to the proof of Theorem 3.17. We need a result about the local behaviour of derivations (see the Appendix). References are [Hei07, Lemma 13.4], [Wea99, Lemma 7.2.3] and [Wea00, Lemma 27]. Note that Proposition 3.16 does not require the measure to be doubling.

Proposition 3.16. Let $A \subset X$ be a measurable set and $D \in \operatorname{Der}(X, \mu)$ a derivation. If the Lipschitz functions $f, g$ agree on $A$, then $D f(x)=D g(x)$ for a.e. $x \in A$.

We can now prove the main result of this section. Note that we assume that the measure $\mu$ is doubling, but the proof just requires the Lebesgue Differentiation Theorem. 
Theorem 3.17. Let $D \in \operatorname{Der}(X, \mu)$ and $f \in \operatorname{Lip}^{\infty}(X)$. Assume that the measure $\mu$ is doubling. Then there is a Borel set $\Omega_{f}$ such that

- $\mu\left(\Omega_{f}\right)=0$;

- if $x \in X \backslash \Omega_{f}$,

$$
|D f(x)| \leq\|D\| £ f(x) .
$$

We will refer to this as the localized derivation inequality.

Proof. Without loss of generality we assume that

$$
\|D\| \leq 1 \text {. }
$$

We apply Lemma 3.7 to $f$ obtaining a Borel partition

$$
X=\bigsqcup_{i=1}^{\infty} A_{i} \sqcup \Omega,
$$

and it sufficies to show that (3.18) holds for a.e. $x \in A_{i}$. We will prove that (3.18) holds if

- $x$ is a Lebesgue point of $D f$, and

- $x$ is a density point of $A_{i}$.

As $£ f$ is continuous on $A_{i}$, it follows that for each $\varepsilon>0$ there is an $r_{0}(x, \varepsilon)>0$ such that if $r \leq r_{0}(x, \varepsilon)$ and $y \in B(x, 2 r)$, then

$$
£ f(y) \leq £ f(x)+\varepsilon ;
$$

as $£ f(\cdot, r) \searrow £ f(\cdot)$ uniformly on $A_{i}$, it follows that for each $\varepsilon>0$ there is an $r_{1}(x, \varepsilon)>0$ such that if $r \leq r_{1}(x, \varepsilon) \leq r_{0}(x, \varepsilon)$, then

$$
£ f(y, 2 r) \leq £ f(y)+\varepsilon .
$$

We now claim that for $r \leq r_{1}(x, \varepsilon)$ the restriction $\left.f\right|_{A_{i} \cap B(x, r)}$ has Lipschitz constant $£ f(x)+2 \varepsilon$. To verify the claim, let $y_{1}, y_{2} \in B(x, r)$. Then $y_{2} \in B\left(y_{1}, 2 r\right)$ and from the definition of $£ f(y, 2 r)$ we conclude that

$$
\left|f\left(y_{1}\right)-f\left(y_{2}\right)\right| \leq £ f(y, 2 r) \rho\left(y_{1}, y_{2}\right) ;
$$

but by (3.20) we conclude that

$$
\left|f\left(y_{1}\right)-f\left(y_{2}\right)\right| \leq(£ f(y)+\varepsilon) \rho\left(y_{1}, y_{2}\right),
$$

and by (3.19) this gives

$$
\left|f\left(y_{1}\right)-f\left(y_{2}\right)\right| \leq(£ f(x)+2 \varepsilon) \rho\left(y_{1}, y_{2}\right),
$$

verifying the claim.

We now note that

$$
\left.(f-f(x))\right|_{A_{i} \cap B(x, r)}
$$

has Lipschitz constant at most $£ f(x)+2 \varepsilon$ and that

$$
\left\|\left.(f-f(x))\right|_{A_{i} \cap B(x, r)}\right\|_{\infty} \leq(£ f(x)+2 \varepsilon) r ;
$$

we can therefore take a MacShane extension $g$ of $\left.(f-f(x))\right|_{A_{i} \cap B(x, r)}$ with

$$
\|g\|_{\operatorname{Lip}^{\infty}(X)} \leq £ f(x)+\varepsilon .
$$

We want to bound

$$
\int_{B(x, r)} D f(y) d \mu(y)
$$


as $r \searrow 0$, because this will give an upper bound for $|D f(x)|$. The product rule implies that $D(1)=0$ a.e., therefore

$$
\int_{B(x, r)} D f(y) d \mu(y)=\int_{B(x, r)} D(f-f(x))(y) d \mu(y) ;
$$

by Proposition $3.16 D(f-f(x))=D g$ a.e. in $A_{i} \cap B(x, r)$ and moreover, as $\|D\| \leq 1$,

$$
|D g| \leq £ f(x)+2 \varepsilon
$$

a.e. Therefore,

$$
\begin{aligned}
\left|f_{B(x, r)} D f(y) d \mu(y)\right|= & \left|\int_{B(x, r)} D(f-f(x))(y) d \mu(y)\right| \\
\leq & \frac{1}{\mu(B(x, r))} \int_{A_{i} \cap B(x, r)}|D g(y)| d \mu(y) \\
& +\frac{1}{\mu(B(x, r))} \int_{B(x, r) \backslash A_{i}}|D(f-f(x))(y)| d \mu(y) \\
\leq & £ f(x)+2 \varepsilon+2\|f\|_{\operatorname{Lip}^{\infty}(X)} \frac{\mu\left(B(x, r) \backslash A_{i}\right)}{\mu(B(x, r))}
\end{aligned}
$$

letting $r \searrow 0$ gives the bound

$$
|D f(x)| \leq £ f(x)+2 \varepsilon .
$$

\section{Measurable differentiable structures}

In this section we recall the definition of measurable differentiable structure. In order to make the exposition more transparent, we decided to first introduce a notion of local independence for Lipschitz functions and, building on this definition, recall Lemma 4.10 which implies the existence of measurable differentiable structures. This Lemma has been either explicitly or implicitly used in previous proofs that a metric measure space admits a measurable differentiable structure [KM11], [Kei04a] and [Che99, Section 4]. The definition of local independence makes also precise the intuitive idea that, if the a space has a differentiable structure, the Lipschitz functions form, infinitesimally, a finite dimensional vector space. To a space possessing a measurable differentiable structure it is possible to associate a natural measurable cotangent bundle. Using the sections of this bundle it is possible to construct Sobolev spaces which are reflexive for $p>1$. In this setting the exterior derivative $d$ extends to Sobolev functions. We finally make an observation relating $d$ and the property that these Sobolev spaces inject into the corresponding $L^{p}$ spaces.

Definition 4.1. (Measurable differentiable structure) A metric measure space $(X, \rho, \mu)$ has a measurable differentiable structure if:

- there is a measurable decomposition

$$
X=\bigcup_{\alpha} X_{\alpha} \cup \Omega
$$

- $\mu(\Omega)=0$ 
- for each set $X_{\alpha}$ there are Lipschitz functions $\left\{x_{\alpha}^{j}\right\}_{j=1}^{N_{\alpha}}$ such that if $f$ is a Lipschitz function, there are unique $\mathrm{L}^{\infty}\left(X_{\alpha}, \mu\right)$ functions

$$
\frac{\partial f}{\partial x_{\alpha}^{j}}: X_{\alpha} \rightarrow \mathbf{R}
$$

such that

$$
£\left\{f-\sum_{j=1}^{N_{\alpha}} \frac{\partial f}{\partial x_{\alpha}^{j}}(x) x_{\alpha}^{j}\right\}(x)=0,
$$

for a.e. $x \in X_{\alpha}$. The pairs $\left(X_{\alpha},\left\{x_{\alpha}^{j}\right\}_{j=1}^{N_{\alpha}}\right)$ are called differentiable charts and the $\frac{\partial f}{\partial x_{\alpha}^{j}}$ are called the partial derivatives of $f$ with respect to the chart functions;

- the integer $N_{\alpha}$ is uniformly bounded. The lowest upper bound is called the dimension of the differentiable structure.

Definition 4.5. (Local independence of Lipschitz functions) Let $f_{1}, \cdots, f_{n}$ be Lipschitz functions. We say that they are independent at $x$ if

$$
£\left(\lambda_{1} f_{1}+\cdots \lambda_{n} f_{n}\right)(x)=0
$$

implies

$$
\lambda_{1}=\cdots=\lambda_{n}=0
$$

where $\lambda_{i} \in \mathbf{R}$.

Another way of thinking of this notion of independence is the following. We can define a map

$$
\begin{aligned}
\Phi_{x}: \mathbf{R}^{n} & \rightarrow \mathbf{R} \\
\left(\lambda_{i}\right) & \mapsto £\left(\sum_{i=1}^{n} \lambda_{i} f_{i}\right)(x) ;
\end{aligned}
$$

from the properties of $£$ we know that $\Phi_{x}$ is a seminorm. The linear independence condition is equivalent to $\Phi_{x}$ being a norm. To establish the existence of a measurable differentiable structure the following principle is usually employed:

Lemma 4.10. Suppose that there is a constant $N$ such that if $\left\{f_{1}, \cdots, f_{n}\right\} \subset$ $\operatorname{Lip}^{\infty}(X)$ are Lipschitz functions which are independent on a set of positive measure $A$, then $n \leq N$. Then $X$ admits a measurable differentiable structure whose dimension is at most $N$.

The proof is a modification of the ideas used to prove Theorem 2.42. The key point is to show that the partial derivatives $\frac{\partial f}{\partial x_{\alpha}^{j}}$ are measurable, and this can be done by using Lemma 2.12. Details can be found in [KM11] and [Kei04a, Section 7.2].

Theorem 4.11. If the metric measure space $(X, \rho, \mu)$ has a measurable differentiable structure, then:

- there exists a measurable cotangent bundle $T^{*} X$;

- on each chart $\left(X_{\alpha},\left\{x_{\alpha}^{j}\right\}_{j=1}^{N_{\alpha}}\right)$ we have a basis $\left\{d x_{\alpha}^{j}\right\}_{j=1}^{N_{\alpha}}$ for the fibres of $T^{*} X_{\alpha}$; 
- we can define a measurable fibrewise norm by setting:

$$
\left\|\left(v_{1}, \cdots, v_{N_{\alpha}}\right)\right\|(x)=£\left\{\sum_{j=1}^{N_{\alpha}} v_{j} x_{\alpha}^{j}\right\}(x) ;
$$

- if on each chart $\left(X_{\alpha},\left\{x_{\alpha}^{j}\right\}_{j=1}^{N_{\alpha}}\right)$, we define

$$
d: \operatorname{Lip}\left(X_{\alpha}\right) \rightarrow \Gamma\left(T^{*} X_{\alpha}\right)
$$

by

$$
d f=\sum_{j=1}^{N_{\alpha}} \frac{\partial f}{\partial x_{\alpha}^{j}} d x_{\alpha}^{j},
$$

then $\|d f\| \in \mathrm{L}^{\infty}\left(X_{\alpha}, \mu\right)$ and

$$
\|d f\|(x)=£ f(x) .
$$

Therefore the set of sections $\Gamma\left(T^{*} X\right)$ is equipped with a norm and we can define $\mathrm{L}^{p}\left(\Gamma\left(T^{*} X\right), \mu\right)$.

The following result is motivated by [Che99, Theorem 4.48]. Note that in this more general setting the Sobolev spaces used by Cheeger might trivially reduce to the corresponding $\mathrm{L}^{p}$ spaces. Therefore, the Sobolev spaces we work with are different from those employed by Cheeger; as far as we understand, the crucial point is that $\mathrm{H}^{1, p}(X, \mu)$ does not need to inject in $\mathrm{L}^{p}(X, \mu)$, so in this setting there is no analogue of the uniqueness statement in [Che99, Theorem 4.47].

Theorem 4.16. If the metric measure space $(X, \rho, \mu)$ has a measurable differentiable structure, define

$$
\mathcal{D}(X, p)=\left\{f \in \operatorname{Lip}(X) \cap \mathrm{L}^{p}(X, \mu): d f \in \mathrm{L}^{p}\left(\Gamma\left(T^{*} X\right), \mu\right)\right\}
$$

and

$$
\|f\|_{\mathrm{H}^{1, p}(X, \mu)}=\|f\|_{\mathrm{L}^{p}(X, \mu)}+\|d f\|_{\mathrm{L}^{p}\left(\Gamma\left(T^{*} X\right), \mu\right)} ;
$$

then $\left(\mathcal{D}(X, p),\|\cdot\|_{\mathrm{H}^{1, p}(X, \mu)}\right)$ is a normed vector space whose completion is denoted by $\mathrm{H}^{1, p}(X, \mu)$ ("Sobolev space"). The space $\mathrm{H}^{1, p}(X, \mu)$ has the following properties:

(1) for $p>1$ the norm $\|\cdot\|_{\mathrm{H}^{1, p}(X, \mu)}$ is equivalent to a uniformly convex norm; in particular, $\mathrm{H}^{1, p}(X, \mu)$ is reflexive;

(2) $\mathrm{H}^{1, p}(X, \mu)$ is isometric to a subspace of the Banach space

$$
\mathrm{L}^{p}(X, \mu) \times \mathrm{L}^{p}\left(\Gamma\left(T^{*} X\right), \mu\right)
$$

whose norm is defined by

$$
\|(f, \gamma)\|_{\mathrm{L}^{p}(X, \mu) \times \mathrm{L}^{p}\left(\Gamma\left(T^{*} X\right), \mu\right)}=\|f\|_{\mathrm{L}^{p}(X, \mu)}+\|\gamma\|_{\mathrm{L}^{p}\left(\Gamma\left(T^{*} X\right), \mu\right)}
$$

(3) there are 1-Lipschitz maps

$$
\begin{aligned}
J: \mathrm{H}^{1, p}(X, \mu) & \rightarrow \mathrm{L}^{p}(X, \mu), \\
(g, \gamma) & \mapsto g ; \\
\tilde{d}: \mathrm{H}^{1, p}(X, \mu) & \rightarrow \mathrm{L}^{p}\left(\Gamma\left(T^{*} X\right), \mu\right), \\
(g, \gamma) & \mapsto \gamma ;
\end{aligned}
$$


which are the restrictions of the projections of $\mathrm{L}^{p}(X, \mu) \times \mathrm{L}^{p}\left(\Gamma\left(T^{*} X\right), \mu\right)$ to $\mathrm{L}^{p}(X, \mu)$ and $\mathrm{L}^{p}\left(\Gamma\left(T^{*} X\right), \mu\right)$, respectively;

(4) $\tilde{d}$ agrees with $d$ on elements $f \in \mathcal{D}(X, p)$ (which we have identified with couples of the form $(f, d f))$.

Proof. By [Che99, Theorem 4.48] we can replace the fibrewise norm $\|\cdot\|_{T^{*} X}$ by a new fibrewise norm $\|\cdot\|_{T^{*} X}^{\prime}$ which is induced, on each fibre, by a scalar product and is equivalent to the previous norm with a bi-Lipschitz constant depending only on the dimension of the measurable differentiable structure. Note that we can regard $\mathcal{D}(X, p)$ as a linear subspace of $\mathrm{L}^{p}(X, \mu) \times \mathrm{L}^{p}\left(\Gamma\left(T^{*} X\right), \mu\right)$ by $f \mapsto(f, d f)$. In particular, taking the closure of this subspace, we conclude that $\mathrm{H}^{1, p}(X, \mu)$ is isometric to a subspace of $\mathrm{L}^{p}(X, \mu) \times \mathrm{L}^{p}\left(\Gamma\left(T^{*} X\right), \mu\right)$, implying $(2)$. The norm $\|\cdot\|_{\mathrm{L}^{p}(X, \mu) \times \mathrm{L}^{p}\left(\Gamma\left(T^{*} X\right), \mu\right)}$ is equivalent to the norm

$$
\|(f, \gamma)\|_{\mathrm{L}^{p}(X, \mu) \times \mathrm{L}^{p}\left(\Gamma\left(T^{*} X\right), \mu\right)}^{\prime}=\left(\int_{X}|f|^{p} d \mu+\int_{X}\left(\|\gamma\|_{T^{*} X}^{\prime}\right)^{p} d \mu\right)^{\frac{1}{p}}
$$

which is uniformly convex, implying (1). Finally (3) follows because we are restricting the projections to the subspace $\mathrm{H}^{1, p}(X, \mu)$ and (4) follows because we identified $f \in$ $\mathcal{D}(X, p)$ with $(f, d f) \in \mathrm{L}^{p}(X, \mu) \times \mathrm{L}^{p}\left(\Gamma\left(T^{*} X\right), \mu\right)$.

Note, however, that if $(f, \gamma) \in \mathrm{H}^{1, p}(X, \mu), f$ does not determine, in general, $\gamma$. For example, let $X$ be a Vitali-Cantor subset of $[0,1]$, i.e. a Cantor subset of $[0,1]$ of positive Lebesgue measure, and $\mu$ the Lebesgue measure; $X$ is constructed similarly to the standard Cantor set but it is not self-similar because the ratio between the size of an interval removed at step $k+1$ and that of an interval removed at step $k$ tends to 0 as $k \rightarrow \infty$. As $X$ is totally disconnected, we can approximate in $\mathrm{L}^{p}(X, \mu)$ any Lipschitz function $f$ on $X$ by functions $f_{n}$ which are Lipschitz and locally constant. In particular, $f_{n} \rightarrow(f, 0)$ in $\mathrm{H}^{1, p}(X, \mu)$. Therefore, both $(f, d f)$ and $(f, 0)$ belong to $\mathrm{H}^{1, p}(X, \mu)$.

We now note that $\mathrm{H}^{1, p}(X, \mu)$ is dense in $\mathrm{L}^{p}(X, \mu)$. The operator $d$ is therefore densely defined in $\mathrm{L}^{p}(X, \mu)$. We recall the following definition from Functional Analysis [Bre11, Chapter 2]:

Definition 4.24. The exterior differential

$$
d: \mathrm{H}^{1, p}(X, \mu) \subset \mathrm{L}^{p}(X, \mu) \rightarrow \mathrm{L}^{p}\left(\Gamma\left(T^{*} X\right), \mu\right)
$$

is said to be a closed operator if $f_{n} \rightarrow f$ in $\mathrm{L}^{p}(X, \mu)$ and $d f_{n} \rightarrow \gamma$ in $\mathrm{L}^{p}\left(\Gamma\left(T^{*} X\right), \mu\right)$ implies that $f \in \mathrm{H}^{1, p}(X, \mu)$ and $d f=\gamma$.

The following Proposition will be used in Section 6 .

Proposition 4.26. The map

$$
J: \mathrm{H}^{1, p}(X, \mu) \rightarrow \mathrm{L}^{p}(X, \mu)
$$

is injective if and only if the operator

$$
d: \mathrm{H}^{1, p}(X, \mu) \subset \mathrm{L}^{p}(X, \mu) \rightarrow \mathrm{L}^{p}\left(\Gamma\left(T^{*} X\right), \mu\right)
$$

is closed.

Proof. Assume that $J$ is injective. Suppose that $f_{n} \rightarrow f$ in $\mathrm{L}^{p}(X, \mu)$ and $d f_{n} \rightarrow$ $\gamma$ in $\mathrm{L}^{p}\left(\Gamma\left(T^{*} X\right), \mu\right)$. Then $\left(f_{n}, d f_{n}\right)$ is a Cauchy sequence in $\mathrm{H}^{1, p}(X, \mu)$ and so it converges to a limit $(f, \gamma)$. As $J$ is injective, $(f, \gamma)=(f, d f)$ showing that $d$ is 
closed. Conversely, assume that $J$ is not injective; we can find $(f, \gamma) \in \mathrm{H}^{1, p}(X, \mu)$ with $\gamma \neq d f$. In particular, there is a sequence of Lipschitz functions $f_{n}$ with $f_{n} \rightarrow f$ in $\mathrm{L}^{p}(X, \mu)$ and $d f_{n} \rightarrow \gamma$ in $\mathrm{L}^{p}(X, \mu)$. As $\gamma \neq d f, d$ is not closed.

\section{Finite dimensionality and derivations}

In this section we prove a finite dimensionality result, that is the existence of a measurable differentiable structure, by assuming an inequality in which the local Lipschitz constant of a function is controlled by finitely many derivations. We have decided to name this inequality (5.11) the reverse infinitesimal derivation inequality. This condition should be compared with the "Lip-derivation" inequality(ies) studied in [Gon11]. One difference is that we allow the constant in the inequality to vary with the point (so we use $\lambda(x)$ ) but independently of the Lipschitz functions. The reverse infinitesimal inequality should also be compared with the "Lip-lip" inequality of [Kei04a]. An explanation about the terminology, "Lip" denotes the local Lipschitz constant $£$ and "lip" the local Lipschitz constant $\ell$. Our argument is based on measure theory and uses linear algebra to imply finite dimensionality. The interplay between measure theory and linear algebra is made possible by an approximation argument, Lemma 5.12, whose proof uses the notion of Lebesgue representative which we now recall.

Definition 5.1. (Lebesgue representative) Let $g \in \mathrm{L}_{\text {loc }}^{1}(X, \mu)$. If the Lebesgue differentiation theorem holds (e.g. if $\mu$ is doubling) we can choose for $g$ the representative defined as follows:

$$
g^{\star}(x)= \begin{cases}\lim _{r}{ } f_{B(x, r)} g(y) d \mu(y) & \text { if the limit exists, } \\ 0 & \text { otherwise. }\end{cases}
$$

In this section if $D$ is a derivation we will use the notation $D^{\star} f$ for the Lebesgue representative of $D f$.

Proposition 5.3. Let $A \subset X, \mu(A)>0$ and $\left\{f_{1}, \cdots, f_{n}\right\} \subset \operatorname{Lip}^{\infty}(A)$. There is a measurable subset $A^{\prime} \subset A$ such that $\mu\left(A \backslash A^{\prime}\right)=0$ and for all $x \in A^{\prime},\left\{c_{1}, \cdots, c_{n}\right\} \subset \mathbf{R}$

$$
D^{\star}\left(\sum_{i=1}^{n} c_{i} f_{i}\right)(x)=\sum_{i=1}^{n} c_{i} D^{\star} f_{i}(x) .
$$

Proof. Let $A^{\prime} \subset A$ be a full measure subset of $A$ such that for each $x \in A^{\prime}$ :

$$
D^{\star} f_{i}(x)=\lim _{r \searrow 0} \int_{B(x, r)} D f_{i}(y) d \mu(y)
$$

if $\left\{c_{1}, \cdots, c_{n}\right\} \subset \mathbf{R}$, then

$$
\lim _{r \searrow 0} \int_{B(x, r)} D\left(\sum_{i=1}^{n} c_{i} f_{i}\right)(y) d \mu(y)=\lim _{r \searrow 0} f_{B(x, r)} \sum_{i=1}^{n} c_{i} D f_{i}(y) d \mu(y) ;
$$

therefore the limit

$$
\lim _{r \searrow 0} \int_{B(x, r)} D\left(\sum_{i=1}^{n} c_{i} f_{i}\right)(y) d \mu(y)
$$


exists and equals

$$
\sum_{i=1}^{n} c_{i} D^{\star} f_{i}(x)
$$

showing that (5.4) holds.

Theorem 5.9. Let $(X, \rho, \mu)$ be a doubling metric measure space. Assume that:

- there are $N$ derivations $D_{1}, \cdots, D_{n}$ and a nowhere vanishing $\lambda \in \mathrm{L}^{\infty}(X, \mu)$;

- for any Lipschitz function $f$, there is a set $\Omega_{f}$ such that

$$
\begin{aligned}
\mu\left(\Omega_{f}\right) & =0 ; \\
\max _{j=1, \cdots, N}\left|D_{j} f(x)\right| & \geq \lambda(x) £ f(x) \quad \forall x \in X \backslash \Omega_{f} ;
\end{aligned}
$$

then $X$ admits of a measurable differentiable structure whose dimension is at most $N$. The relation (5.11) will be referred to as the reverse infinitesimal derivation inequality.

As we already said, the proof relies on the following approximation argument. The point is that if we have a linear dependence relation where the $c_{i}$ are functions, we would like to treat them as constants so that the linear dependence relation "localizes" at the points of a full measure subset.

Lemma 5.12. Assume that the derivation inequality (5.11) holds. Let $A \subset X$, $\mu(A)>0$ and $\left\{f_{1}, \cdots, f_{n}\right\} \subset \operatorname{Lip}^{\infty}(A)$. There is a measurable subset $A^{\prime} \subset A$ such that $\mu\left(A \backslash A^{\prime}\right)=0$ and for all $x \in A^{\prime},\left\{c_{1}, \cdots, c_{n}\right\} \subset \mathbf{R}$

$$
\begin{aligned}
& \max _{j=1, \cdots, N}\left|D_{j}^{\star}\left(\sum_{i=1}^{n} c_{i} f_{i}\right)(x)\right| \geq \lambda(x) £\left(\sum_{i=1}^{n} c_{i} f_{i}\right)(x) \\
&\left|D_{j}^{\star}\left(\sum_{i=1}^{n} c_{i} f_{i}\right)(x)\right| \leq\left\|D_{j}\right\| £\left(\sum_{i=1}^{n} c_{i} f_{i}\right)(x) \quad \text { for } j=1, \cdots, N .
\end{aligned}
$$

Proof. Let $\Psi \subset S^{n-1}$ be a countable dense subset of the unit sphere and let

$$
\Psi\left(f_{1}, \cdots, f_{n}\right) \equiv\left\{\sum_{i=1}^{n} a_{i} f_{i}:\left(a_{1}, \cdots, a_{n}\right) \in \Psi\right\} .
$$

Given a function $f \in \operatorname{Lip}^{\infty}(A)$ let $\Omega_{f}$ denote the set where either one of the followings fails:

$$
\begin{aligned}
\max _{j=1, \cdots, N}\left|D_{j}^{\star} f(x)\right| & \geq \lambda(x) £ f(x) \\
\left|D_{j}^{\star} f(x)\right| & \leq\left\|D_{j}\right\| £ f(x) \quad \text { for } j=1, \cdots, N ;
\end{aligned}
$$

by assumption and by Theorem $3.17, \mu\left(\Omega_{f}\right)=0$. Let

$$
\Omega_{\Psi}\left(f_{1}, \cdots, f_{n}\right)=\bigcup_{f \in \Psi\left(f_{1}, \cdots, f_{n}\right)} \Omega_{f}
$$

then for $x \in A \backslash \Omega_{\Psi}\left(f_{1}, \cdots, f_{n}\right)$, (5.16) and (5.17) hold for any multiple $c f$ with $f \in \Psi\left(f_{1}, \cdots, f_{n}\right)$ and $c \in \mathbf{R}$. Let us fix some $\left\{c_{1}, \cdots, c_{n}\right\} \subset \mathbf{R}$; for any $\varepsilon>0$ there is a

$$
\left(b_{1}, \cdots, b_{n}\right)=b \cdot\left(a_{1}, \cdots, a_{n}\right)
$$


such that

$$
\begin{aligned}
& \left(a_{1}, \cdots, a_{n}\right) \in \Psi \\
& \sum_{i=1}^{n}\left|c_{i}-b_{i}\right| \leq \varepsilon
\end{aligned}
$$

in particular

$$
\begin{aligned}
\max _{j=1, \cdots, N}\left|D_{j}^{\star}\left(\sum_{i=1}^{n} b_{i} f_{i}\right)(x)\right| & \geq \lambda(x) £\left(\sum_{i=1}^{n} b_{i} f_{i}\right)(x) \\
\left|D_{j}^{\star}\left(\sum_{i=1}^{n} b_{i} f_{i}\right)(x)\right| & \leq\left\|D_{j}\right\| £\left(\sum_{i=1}^{n} b_{i} f_{i}\right)(x) \text { for } j=1, \cdots, N .
\end{aligned}
$$

Let

$$
\begin{aligned}
& C_{1}=\bigvee\left\|f_{i}\right\|_{\operatorname{Lip}^{\infty}(X)} \\
& C_{2}=\bigvee\left\|D_{j}\right\| ;
\end{aligned}
$$

then

$$
\left|D_{j}^{\star} f_{i}(x)\right| \leq C_{1} C_{2}
$$

We now make two estimates:

$$
£\left(\sum_{i=1}^{n}\left(c_{i}-b_{i}\right) f_{i}\right)(x) \leq \sum_{i=1}^{n}\left|c_{i}-b_{i}\right| £ f_{i}(x) \leq \varepsilon C_{1} ;
$$

and

$$
\begin{aligned}
\left|D_{j}^{\star}\left(\sum_{i=1}^{n} b_{i} f_{i}\right)(x)-D_{j}^{\star}\left(\sum_{i=1}^{n} c_{i} f_{i}\right)(x)\right| & =\left|\sum_{i=1}^{n}\left(b_{i}-c_{i}\right) D_{j}^{\star} f_{i}(x)\right| \\
& \leq \sum_{i=1}^{n}\left|b_{i}-c_{i}\right|\left|D_{j}^{\star} f_{i}(x)\right| \\
& \leq \varepsilon C_{1} C_{2} .
\end{aligned}
$$

Substitution of the last two estimates into (5.22) and (5.23) leads to

$$
\begin{aligned}
\max _{j=1, \cdots, N}\left|D_{j}^{\star}\left(\sum_{i=1}^{n} c_{i} f_{i}\right)(x)\right| \geq \lambda(x) £\left(\sum_{i=1}^{n} c_{i} f_{i}\right)(x)-\varepsilon C_{1} C_{2}-\varepsilon C_{1} \lambda(x) ; \\
\left|D_{j}^{\star}\left(\sum_{i=1}^{n} c_{i} f_{i}\right)(x)\right| \leq\left\|D_{j}\right\| £\left(\sum_{i=1}^{n} c_{i} f_{i}\right)(x)+2 \varepsilon C_{1} C_{2}
\end{aligned}
$$

for $j=1, \cdots, N$. Letting $\varepsilon \searrow 0$ completes the proof of (5.13) and (5.14).

Proof of Theorem 5.9. The proof is reduced to Proposition 4.10. We assume that there are $n$ Lipschitz functions $\left\{f_{1}, \cdots, f_{n}\right\} \subset \operatorname{Lip}(X)$ which are independent at each point $x \in A$, where $\mu(A)>0$. We show that $n \leq N$ arguing by contrapositive: we show that if $n \geq N$ then the functions $\left\{f_{1}, \cdots, f_{n}\right\}$ are dependent on a positive 
measure subset of $A$. Without loss of generality we can assume that $A$ is bounded and replace each $f_{i}$ by

$$
\left(f_{i} \wedge \sup _{A}\left|f_{i}\right|\right) \vee\left(-\sup _{A}\left|f_{i}\right|\right)
$$

so that

$$
\left\{f_{1}, \cdots, f_{n}\right\} \subset \operatorname{Lip}^{\infty}(X) .
$$

As $\lambda$ is nowhere vanishing, we can suppose that $\lambda \geq C>0$ on $A$. By Lemma 5.12 there is $A^{\prime} \subset A$ such that $\mu\left(A \backslash A^{\prime}\right)=0$ and (5.13) and (5.14) hold on $A^{\prime}$ (with $\lambda$ replaced by $C$ ):

$$
\begin{aligned}
\max _{j=1, \cdots, N}\left|D_{j}^{\star}\left(\sum_{i=1}^{n} c_{i} f_{i}\right)(x)\right| & \geq C £\left(\sum_{i=1}^{n} c_{i} f_{i}\right)(x) \\
\left|D_{j}^{\star}\left(\sum_{i=1}^{n} c_{i} f_{i}\right)(x)\right| & \leq\left\|D_{j}\right\| £\left(\sum_{i=1}^{n} c_{i} f_{i}\right)(x) \text { for } j=1, \cdots, N .
\end{aligned}
$$

Let us consider the matrix

$$
F=\left(\begin{array}{ccc}
D_{1}^{\star} f_{1} & \cdots & D_{1}^{\star} f_{n} \\
\vdots & \cdots & \vdots \\
D_{N} \star f_{1} & \cdots & D_{N}^{\star} f_{n}
\end{array}\right),
$$

with entries in $\mathrm{L}^{\infty}\left(A^{\prime}, \mu\right)$. Since $n>N$, there is a measurable $B \subset A^{\prime}$ with $\mu(B)>0$ and $\operatorname{rank} F(x)=k<n$ for $x \in B$. Without loss of generality, we can assume that the first $k$ columns of $F$ are linearly independent on $B$ and the first $k+1$ columns of $F$ are linearly dependent on $B$. By Lemma 2.12 there are $\lambda_{i} \in \mathrm{L}^{\infty}(B, \mu)$ with

$$
\mu\left(\left\{x \in B: \forall i, \lambda_{i}(x)=0\right\}\right)=0 .
$$

and

$$
\sum_{i=1}^{k+1} \lambda_{i}(x)\left(D_{j}^{\star} f_{i}\right)(x)=0,
$$

for all $x \in B$ and all $j=1, \cdots, N$. We now choose $x \in B$ and define $c_{i}=\lambda_{i}(x)$. By Proposition 5.3 we have

$$
\left|D_{j}^{\star}\left(\sum_{i=1}^{k+1} c_{i} f_{i}\right)(x)\right|=0 \quad \text { for } j=1, \cdots, N
$$

and by (5.33)

$$
£\left(\sum_{i=1}^{k+1} c_{i} f_{i}\right)(x)=0 .
$$

So the $\left\{f_{1}, \cdots, f_{n}\right\}$ are dependent at a.e. $x \in B$. 


\section{Choice of the chart functions}

In this section we present some results connected with the choice of the chart functions. The starting point is the representation formula (6.3) for derivations if the space admits a measurable differentiable structure. This formula has an interesting consequence: if the partial derivatives are known to be derivations, they give a basis for the module of derivations of the chart (Corollary 6.15). This naturally leads to the following question: when are the partial derivatives derivations? We have found two sufficient conditions but we have been unable to find a complete answer. If the answer were negative, then there would be two kinds of differentiable structures and those in which the partial derivatives are also derivations would exhibit a more regular behaviour. We next investigate the choice of the chart functions generalizing the results of [Kei04b]. The main result is that, knowing that the partial derivatives are derivations, the chart functions can be chosen among a generating set for the Lipschitz algebra. This implies immediately that the chart functions can be chosen among distance functions if the metric space $X$ is bounded. Noting that we can always assume the charts to be bounded by partitioning them, this implies that chart functions can be chosen among distance functions. Note that in general the distance functions have to be chosen as distance functions from points of each chart. For example, let $\left(U_{1}, \rho_{1}, \mu_{1}\right)$ and $\left(U_{2}, \rho_{2}, \mu_{2}\right)$ be differentiability spaces (of positive dimension) of diametre at most 1 . The metric space $\left(U_{1} \sqcup U_{2}, \rho\right)$ is defined by letting

$$
\rho(u, v)= \begin{cases}\rho_{i}(u, v) & \text { if } u, v \in U_{i} \\ 1 & \text { otherwise }\end{cases}
$$

The metric measure space $\left(U_{1} \sqcup U_{2}, \rho, \mu_{1}+\mu_{2}\right)$ is a differentiability space and all distance functions from points of $U_{1}$ are constant on $U_{2}$, so their differentials vanish and these functions cannot be chosen as chart functions.

Lemma 6.2. Suppose the doubling metric measure space $(X, \rho, \mu)$ has a measurable differentiable structure, and let $\left(X_{\alpha},\left\{x_{\alpha}^{j}\right\}_{j=1}^{N_{\alpha}}\right)$ be a chart with $\left\{x_{\alpha}^{j}\right\}_{j=1}^{N_{\alpha}} \subset$ $\operatorname{Lip}^{\infty}\left(X_{\alpha}\right)$. If $D \in \operatorname{Der}\left(X_{\alpha}, \mu\right)$ and $f \in \operatorname{Lip}^{\infty}\left(X_{\alpha}\right)$, then

$$
D f=\sum_{j=1}^{N_{\alpha}} \frac{\partial f}{\partial x_{\alpha}^{j}} D x_{\alpha}^{j} .
$$

Before giving the proof of the Lemma we will restate part of Lemma 5.12 and of Proposition 5.3. The point is that in the proof of Lemma 5.12 the proofs of the statements of (5.13) and (5.14) are indepedent. While (5.13) depends on the reverse infinitesimal derivation inequality (5.11), (5.14) is just a consequence of the localized derivation inequality (3.17) (which is true in a doubling metric space or, more generally, in any metric measure space where the Lebesgue Differentiation Theorem holds).

Lemma 6.4. Let $A \subset X$ be a measurable subset of positive measure, let

$$
\left\{f_{1}, \cdots, f_{n}\right\} \subset \operatorname{Lip}^{\infty}(A)
$$

and let

$$
\left\{D_{1}, \cdots, D_{N}\right\} \subset \operatorname{Der}(A, \mu)
$$


There is a measurable subset $A^{\prime} \subset A$ such that $\mu\left(A \backslash A^{\prime}\right)=0$ and for all $x \in A^{\prime}$, $\left\{c_{1}, \cdots, c_{n}\right\} \subset \mathbf{R}$

$$
\begin{aligned}
&\left|D_{j} \star\left(\sum_{i=1}^{n} c_{i} f_{i}\right)(x)\right| \leq\left\|D_{j}\right\| £\left(\sum_{i=1}^{n} c_{i} f_{i}\right)(x) \quad \text { for } j=1, \cdots, N . \\
& D_{j}^{\star}\left(\sum_{i=1}^{n} c_{i} f_{i}\right)(x)=\sum_{i=1}^{n} c_{i} D_{j}^{\star} f_{i}(x) \quad \text { for } j=1, \cdots, N .
\end{aligned}
$$

Proof of Lemma 6.2. Without loss of generality we assume that $X_{\alpha}$ is bounded, $\mu\left(X_{\alpha}\right)<\infty$. We will show that given $f \in \operatorname{Lip}^{\infty}\left(X_{\alpha}\right)$ and $D \in \operatorname{Der}\left(X_{\alpha}, \mu\right)$, there is a measurable subset $C_{f, D} \subset X_{\alpha}$ with $\mu\left(X_{\alpha} \backslash C_{f, D}\right)=0$ and for all $z \in C_{f, D}$,

$$
D^{\star} f(z)=\sum_{j=1}^{N_{\alpha}} \frac{\partial f}{\partial x_{\alpha}^{j}}(z) D^{\star} x_{\alpha}^{j}(z) .
$$

This will imply (6.3). We apply Lemma 6.4 with $A=X_{\alpha}$,

$$
\left\{D_{1}, \cdots, D_{N}\right\}=\{D\},
$$

and

$$
\left\{f_{1}, \cdots, f_{n}\right\}=\left\{f, x_{\alpha}^{1}, \cdots, x_{\alpha}^{N_{\alpha}}\right\},
$$

to obtain a measurable subset $A_{f, D} \subset X_{\alpha}$ such that

- $\mu\left(X_{\alpha} \backslash A_{f, D}\right)=0$,

- for all $z \in A_{f, D},\left\{a, c_{1}, \cdots, c_{N_{\alpha}}\right\} \subset \mathbf{R}$,

$$
\begin{aligned}
\left|D^{\star}\left(a f+\sum_{j=1}^{N_{\alpha}} c_{j} x_{\alpha}^{j}\right)(z)\right| & \leq\|D\| £\left(a f+\sum_{j=1}^{N_{\alpha}} c_{j} x_{\alpha}^{j}\right)(z) . \\
D^{\star}\left(a f+\sum_{j=1}^{N_{\alpha}} c_{j} x_{\alpha}^{j}\right)(z) & =a D^{\star} f(z)+\sum_{j=1}^{N_{\alpha}} c_{j} D^{\star} x_{\alpha}^{j}(z) .
\end{aligned}
$$

From the definition of measurable differentiable structure there are a measurable subset $B_{f, D} \subset X_{\alpha}$ and maps:

such that

$$
\frac{\partial f}{\partial x_{\alpha}^{j}}: B_{f, D} \rightarrow \mathbf{R} \quad \text { for } j=1, \cdots, N_{\alpha}
$$

- $\mu\left(X_{\alpha} \backslash B_{f, D}\right)=0$;

- $\forall z \in B_{f, D}$

$$
\sup _{j=1, \cdots, N_{\alpha}} \sup _{z \in B_{f, D}}\left|\frac{\partial f}{\partial x_{\alpha}^{j}}(z)\right| \leq C \mathbf{L}(f),
$$

- $\forall z \in B_{f, D}$

$$
£\left(f-\sum_{j=1}^{N_{\alpha}} \frac{\partial f}{\partial x_{\alpha}^{j}}(z) x_{\alpha}^{j}\right)(z)=0 .
$$

If we let $C_{f, D}=A_{f, D} \cap B_{f, D}$, set

$$
\left(a, c_{1}, \cdots, c_{N_{\alpha}}\right)=\left(-1, \frac{\partial f}{\partial x_{\alpha}^{1}}(z), \cdots, \frac{\partial f}{\partial x_{\alpha}^{N_{\alpha}}}(z)\right)
$$


in (6.8), apply (6.10) and finally use (6.9) we deduce (6.7).

Corollary 6.11. Suppose the doubling metric measure space $(X, \rho, \mu)$ has a measurable differentiable structure which has dimension $N$. Then $\operatorname{Der}(X, \mu)$ has rank locally bounded by $N$, in particular Theorem 2.42 applies.

Proof. As the charts measurably partition $X$, it suffices to show that if $U \subset X_{\alpha}$ has positive measure and if the derivations

$$
\left\{D_{1}, \cdots, D_{n}\right\} \subset \operatorname{Der}(U, \mu)
$$

are linearly independent, then $n \leq N_{\alpha} \leq N$. We argue by contrapositive, that is, by showing that if $n>N_{\alpha}$, the derivations cannot be linearly independent. Using Lemma 6.4 we find a set $U^{\prime} \subset U$ with $\mu\left(U \backslash U^{\prime}\right)=0$ and (5.33) and (6.5) hold for the derivations $\left\{D_{1}, \cdots, D_{n}\right\}$ and the chart functions. We now consider the matrix

$$
F=\left(\begin{array}{ccc}
D_{1}^{\star} x_{\alpha}^{1} & \cdots & D_{1}^{\star} x_{\alpha}^{N_{\alpha}} \\
\vdots & \cdots & \vdots \\
D_{n}^{\star} x_{\alpha}^{1} & \cdots & D_{n}^{\star} x_{\alpha}^{N_{\alpha}}
\end{array}\right)
$$

with entries in $\mathrm{L}^{\infty}\left(U^{\prime}, \mu\right)$. As $n>N_{\alpha}$ there is a measurable subset $U^{\prime \prime} \subset U^{\prime}$ of positive measure on which the rank of $F$ is $k<n$. Without loss of generality we can assume that the first $k$ rows are linearly independent and the first $k+1$ rows are linearly dependent. By Lemma 2.12 there are $k+1$ functions $\lambda_{i} \in \mathrm{L}^{\infty}\left(U^{\prime \prime}, \mu\right)$ such that

$$
\begin{aligned}
\left\|\lambda_{i}\right\|_{L^{\infty}(A, \mu)} & \leq 1, \\
\sum_{i=1}^{k+1} \lambda_{i}(x) D_{i}^{\star} x_{\alpha}^{j}(z) & =0 \quad \text { for a.e. } z \in U^{\prime \prime} \text { and } j=1, \cdots, N_{\alpha}, \\
\mu\left(\left\{x: \forall i, \lambda_{i}(x)=0\right\}\right) & =0 .
\end{aligned}
$$

From (6.3) we deduce that

$$
\sum_{i=1}^{k+1} \lambda_{i} D_{i}^{\star}=0
$$

in $\operatorname{Der}\left(U^{\prime \prime}, \mu\right)$ showing that the derivations $\left\{D_{1}, \cdots, D_{k+1}\right\}$ are not linearly independent.

Corollary 6.15. Suppose the doubling metric measure space $(X, \rho, \mu)$ has a measurable differentiable structure, and let $\left(X_{\alpha},\left\{x_{\alpha}^{j}\right\}_{j=1}^{N_{\alpha}}\right)$ be a chart. If the partial derivatives $\left\{\frac{\partial}{\partial x_{\alpha}^{j}}\right\}_{j=1}^{N_{\alpha}} \subset \operatorname{Der}\left(X_{\alpha}, \mu\right)$, then $\operatorname{Der}\left(X_{\alpha}, \mu\right)$ is free and $\left\{\frac{\partial}{\partial x_{\alpha}^{j}}\right\}_{j=1}^{N_{\alpha}}$ is a basis.

We now give two criteria for the "partial derivatives" to be derivations.

Lemma 6.16. Suppose the doubling metric measure space $(X, \rho, \mu)$ has a measurable differentiable structure, and let $\left(X_{\alpha},\left\{x_{\alpha}^{j}\right\}_{j=1}^{N_{\alpha}}\right)$ be a chart. If the map

$$
J: \mathrm{H}^{1, p}(X, \mu) \rightarrow \mathrm{L}^{p}(X, \mu)
$$


is injective, then the maps

$$
\begin{gathered}
\frac{\partial}{\partial x_{\alpha}^{j}}: \operatorname{Lip}^{\infty}\left(X_{\alpha}\right) \rightarrow \mathrm{L}^{\infty}\left(X_{\alpha}, \mu\right), \\
f \mapsto \frac{\partial f}{\partial x_{\alpha}^{j}}
\end{gathered}
$$

are derivations.

Proof. Recall Definition 2.5 where the axioms that derivations have to satisfy are stated. The partial derivatives $\frac{\partial}{\partial x_{\alpha}^{j}}$ satisfy linearity, boundedness and the product rule by definition. We have to check weak* continuity. As usual, there is no loss of generality in assuming that $X_{\alpha}$ is bounded and of finite measure. Let $\left\{f_{k}\right\} \subset$ $\operatorname{Lip}^{\infty}\left(X_{\alpha}\right)$ and $f_{k} \rightarrow f$ weak* in $\operatorname{Lip}^{\infty}\left(X_{\alpha}\right)$, that is, $f_{k} \rightarrow f$ uniformly with $\mathbf{L}\left(f_{k}\right)$ uniformly bounded. We have to show that

$$
\frac{\partial f_{k}}{\partial x_{\alpha}^{j}} \rightarrow \frac{\partial f}{\partial x_{\alpha}^{j}}
$$

weak ${ }^{*}$ in $\mathrm{L}^{\infty}\left(X_{\alpha}, \mu\right)$. We will prove the following statement: for any subsequence $\left\{f_{k_{l}}\right\} \subset\left\{f_{k}\right\}$ we can pass to a further subsequence $\left\{f_{\tilde{k}_{l}}\right\} \subset\left\{f_{k_{l}}\right\}$ such that

$$
\frac{\partial f_{\tilde{k}_{l}}}{\partial x_{\alpha}^{j}} \rightarrow \frac{\partial f}{\partial x_{\alpha}^{j}}
$$

weak* in $\mathrm{L}^{\infty}\left(X_{\alpha}, \mu\right)$. This means that for any $g \in \mathrm{L}^{1}\left(X_{\alpha}, \mu\right)$ we have to show that

$$
\int_{X_{\alpha}} \frac{\partial f_{\tilde{k}_{l}}}{\partial x_{\alpha}^{j}} g d \mu \rightarrow \int_{X_{\alpha}} \frac{\partial f}{\partial x_{\alpha}^{j}} g d \mu .
$$

As the sequence $\frac{\partial f_{k}}{\partial x_{\alpha}^{j}}$ is uniformly bounded in $\mathrm{L}^{\infty}\left(X_{\alpha}, \mu\right)$ and as continuous functions are dense in $\mathrm{L}^{1}\left(X_{\alpha}, \mu\right)$, it will suffice to consider $g$ continuous in (6.19). We observe that $\left\{f_{k_{l}}\right\}$ is a bounded sequence in $\mathrm{H}^{1, p}\left(X_{\alpha}, \mu\right)$ and by reflexivity we can pass to a subsequence $\left\{f_{\tilde{k}_{l}}\right\}$ such that $f_{\tilde{k}_{l}} \rightarrow h$ weakly in $\mathrm{H}^{1, p}\left(X_{\alpha}, \mu\right)$. As $\mathrm{H}^{1, p}\left(X_{\alpha}, \mu\right)$ biLipschitz embedds in $\mathrm{L}^{p}(X, \mu) \times \mathrm{L}^{p}\left(\Gamma\left(T^{*} X\right), \mu\right)$, we conclude that $h=(f, \gamma)$ and $d f_{\tilde{k}_{l}} \rightarrow \gamma$ weakly in $\mathrm{L}^{p}\left(\Gamma\left(T^{*} X\right), \mu\right)$. This implies that for every continuous function $g$,

$$
\int_{X_{\alpha}} \frac{\partial f_{\tilde{k}_{l}}}{\partial x_{\alpha}^{j}} g d \mu \rightarrow \int_{X_{\alpha}} \gamma_{j} g d \mu,
$$

but, as $J$ is injective, $\gamma=d f$ showing that (6.19) holds.

Lemma 6.20. Suppose the doubling metric measure space $(X, \rho, \mu)$ satisfies the hypotheses of Theorem 5.9 (in particular the reverse infinitesimal derivation inequality), and let $\left(X_{\alpha},\left\{x_{\alpha}^{j}\right\}_{j=1}^{N_{\alpha}}\right)$ be a chart. Then the maps

$$
\begin{gathered}
\frac{\partial}{\partial x_{\alpha}^{j}}: \operatorname{Lip}^{\infty}\left(X_{\alpha}\right) \rightarrow \mathrm{L}^{\infty}\left(X_{\alpha}, \mu\right), \\
f \mapsto \frac{\partial f}{\partial x_{\alpha}^{j}}
\end{gathered}
$$

are derivations. 
Proof. Let us consider the matrix

$$
F=\left(\begin{array}{ccc}
D_{1}^{\star} x_{\alpha}^{1} & \cdots & D_{1}^{\star} x_{\alpha}^{N_{\alpha}} \\
\vdots & \cdots & \vdots \\
D_{N}^{\star} x_{\alpha}^{1} & \cdots & D_{N}^{\star} x_{\alpha}^{N_{\alpha}}
\end{array}\right)
$$

with entries in $\mathrm{L}^{\infty}\left(X_{\alpha}, \mu\right)$. We first show that this matrix has a.e. rank $N_{\alpha}$. Suppose on the contrary that on some subset $U \subset X_{\alpha}$ with $\mu(U)>0$ the rank of $F$ is $k<N_{\alpha}$. Without loss of generality we can assume that the first $k$ columns are independent while the first $k+1$ columns are linearly dependent. By Lemma 2.12 there are $k+1$ functions $\lambda_{i} \in \mathrm{L}^{\infty}(U, \mu)$ such that

$$
\begin{aligned}
\left\|\lambda_{i}\right\|_{L^{\infty}(U, \mu)} & \leq 1, \\
\sum_{i=1}^{k+1} \lambda_{i}(x) D_{j}^{\star} x_{\alpha}^{i} & =0 \quad \text { for a.e. } z \in U \text { and } j=1, \cdots, N, \\
\mu\left(\left\{x: \forall i, \lambda_{i}(x)=0\right\}\right) & =0 .
\end{aligned}
$$

We choose a subset $U^{\prime} \subset U$ with $\mu\left(U^{\prime} \backslash U\right)$ and such that the conclusions of Proposition 5.3 and Lemma 5.12 hold for the derivations $\left\{D_{1}, \cdots, D_{N}\right\}$ and the chart functions $\left\{x_{\alpha}^{1}, \cdots, x_{\alpha}^{k+1}\right\}$. For $z \in U^{\prime}$ application of (5.13) for

$$
\left(c_{1}, \cdots, c_{k+1}\right)=\left(\lambda_{1}(z), \cdots, \lambda_{k+1}(z)\right)
$$

shows that the chart functions $\left\{x_{\alpha}^{1}, \cdots, x_{\alpha}^{k+1}\right\}$ are dependent at $z$, leading to a contradiction. Therefore, the rank of $F$ is a.e. $N_{\alpha}$. So given $U \subset X_{\alpha}$ of positive measure we can find $V \subset U$ of positive measure and an $N_{\alpha} \times N_{\alpha}$ minor of $F$ whose determinant does not vanish on $V$. Without loss of generality we will assume that

$$
G=\left(\begin{array}{ccc}
D_{1}^{\star} x_{\alpha}^{1} & \cdots & D_{1}^{\star} x_{\alpha}^{N_{\alpha}} \\
\vdots & \cdots & \vdots \\
D_{N_{\alpha}}^{\star} x_{\alpha}^{1} & \cdots & D_{N_{\alpha}}^{\star} x_{\alpha}^{N_{\alpha}}
\end{array}\right)
$$

is non-singular on $V$. Using an argument similar to that of Corollary 2.30 we can find $V^{\prime} \subset V$ with $\mu\left(V^{\prime}\right)>0$ and derivations $\left\{D_{1}^{\prime}, \cdots, D_{N_{\alpha}}^{\prime}\right\} \subset \operatorname{Der}\left(V^{\prime}, \mu\right)$ such that

$$
D_{i}^{\prime} x_{\alpha}^{j}=\delta_{i}^{j}
$$

This shows that the maps

$$
\chi_{V^{\prime}} \frac{\partial}{\partial x_{\alpha}^{j}}
$$

are derivations (here we use the Representation Formula (6.3)). Therefore for any $U \subset X_{\alpha}$ of positive measure, there is a subset $V \subset U$ of positive measure such that the

$$
\chi_{V} \frac{\partial}{\partial x_{\alpha}^{j}}
$$

are derivations. Using an exhaustion argument similar to that in the proof of Theorem 2.42 we find a measurable partition

$$
X_{\alpha}=\bigsqcup_{i} V_{i} \sqcup \Omega
$$


with $\mu(\Omega)=0$ and each

$$
\chi_{V_{i}} \frac{\partial}{\partial x_{\alpha}^{j}}
$$

is a derivation $\left(\right.$ in $\left.\operatorname{Der}\left(X_{\alpha}, \mu\right)\right)$. Then the

$$
\frac{\partial}{\partial x_{\alpha}^{j}}=\sum_{i} \chi_{V_{i}} \frac{\partial}{\partial x_{\alpha}^{j}}
$$

are derivations on the disjoint union $\bigsqcup_{i} V_{i}$.

In the next theorem we prove that chart functions can be chosen among a generating set for the Lipschitz algebra.

Theorem 6.26. Suppose the doubling metric measure space $(X, \rho, \mu)$ admits a measurable differentiable structure and that for each chart $\left(X_{\alpha},\left\{x_{\alpha}^{j}\right\}_{j=1}^{N_{\alpha}}\right)$ the partial derivatives are derivations. If $\left\{g_{j}\right\}_{j=1}^{M}$ is a generating set for the Lipschitz algebra $\operatorname{Lip}^{\infty}(X)$, the charts can be chosen so that the chart functions belong to $\left\{g_{j}\right\}_{j=1}^{M}$.

Proof. From Corollary 6.15 we know that $\left\{\frac{\partial}{\partial x_{\alpha}^{j}}\right\}$ is a basis for $\operatorname{Der}\left(X_{\alpha}, \mu\right)$. Given any $U \subset X_{\alpha}$ with $\mu(U)>0$ we can apply Corollary 2.30 to find $V \subset U$ with $\mu(V)>0$, functions $\left\{g_{1}^{\prime}, \cdots, g_{N_{\alpha}}^{\prime}\right\} \subset\left\{g_{j}\right\}_{j=1}^{M}$ and derivations $\left\{D_{1}^{\prime}, \cdots, D_{N_{\alpha}}^{\prime}\right\} \subset \operatorname{Der}(V, \mu)$ such that

$$
D_{i}^{\prime} g_{j}^{\prime}=\delta_{i, j}
$$

Applying Lemma 6.4 to $\left\{D_{1}^{\prime}, \cdots, D_{N_{\alpha}}^{\prime}\right\}$ and $\left\{g_{1}^{\prime}, \cdots, g_{N_{\alpha}}^{\prime}\right\}$ we find $V^{\prime} \subset V$ with $\mu\left(V \backslash V^{\prime}\right)=0$ and for each $z \in V^{\prime}$,

$$
\begin{aligned}
\max _{i=1, \cdots, N_{\alpha}}\left|c_{i}\right| & =\max _{j=1, \cdots, N_{\alpha}}\left|D_{j}^{\prime \star}\left(\sum_{i=1}^{N_{\alpha}} c_{i} g_{i}^{\prime}\right)(z)\right| \\
& \leq \max _{j=1, \cdots, N_{\alpha}}\left\|D_{j}^{\prime}\right\| £\left(\sum_{i=1}^{N_{\alpha}} c_{i} g_{i}^{\prime}\right)(z),
\end{aligned}
$$

which shows that the functions $\left\{g_{1}^{\prime}, \cdots, g_{N_{\alpha}}^{\prime}\right\}$ are a.e. independent on $V$, implying that $\left(V,\left\{g_{i}^{\prime}\right\}_{i=1}^{N_{\alpha}}\right)$ is a chart. Using and exhaustion argument similar to that in the proof of Theorem 2.42 we can "cover" (up to a subset of measure 0) $X_{\alpha}$ by measurable charts such that the chart functions are among the $\left\{g_{j}\right\}_{j=1}^{M}$.

The classical Stone-Weierstrass Theorem pertains to the Banach Algebra $C(X)$ of continuous functions on a compact space $X$, where the norm is the sup-norm. There is an analogue of this result in the setting of Lipschitz algebras. From now to the end of this section we will assume that $X$ is bounded. The condition of separating points is replaced by an uniform condition:

Definition 6.28. A subalgebra $\mathcal{A} \subset \operatorname{Lip}^{\infty}(X)$ is said to separate points uniformly if there is a constant $M>0$ such that for any pair of points $x_{1}, x_{2} \in X$ there is an $f \in \mathcal{A}$ such that

$$
\left|f\left(x_{1}\right)-f\left(x_{2}\right)\right|=\rho\left(x_{1}, x_{2}\right) \quad \text { and } \quad\|f\|_{\operatorname{Lip}^{\infty}(X)} \leq M .
$$

We state the result for $\operatorname{Lip}^{\infty}(X)$, in [Wea99, Chapter 4] one can find the proof for $\operatorname{Lip}_{0}\left(X, x_{0}\right)$. 
Theorem 6.30. (Stone-Weierstrass for Lipschitz algebras) If $\mathcal{A} \subset \operatorname{Lip}^{\infty}(X)$ is a weak* closed subalgebra (with the same unit as $\operatorname{Lip}^{\infty}(X)$ ) that separates points uniformly, then $\mathcal{A}=\operatorname{Lip}^{\infty}(X)$.

Corollary 6.31. Under the hypotheses of Theorem 6.26 the chart functions can be chosen among distance functions from points.

Proof. By intersection with balls of increasing radii, any chart can be partitioned into bounded charts $X_{\alpha}$. As a consequence of the Stone-Weierstrass Theorem (6.30), the distance functions from points are a generating set for $\operatorname{Lip}^{\infty}\left(X_{\alpha}\right)$ and hence the chart functions can be chosen among distance functions.

\section{References}

[AE56] Arens, R. F., and J. Eells, JR.: On embedding uniform and topological spaces. - Pacific J. Math. 6, 1956, 397-403.

[Bre11] Brezis, H.: Functional analysis, Sobolev spaces and partial differential equations. - Universitext, Springer, New York, 2011.

[Che99] Cheeger, J.: Differentiability of Lipschitz functions on metric measure spaces. - Geom. Funct. Anal. 9:3, 1999, 428-517.

[Gon11] Gong, J.: Differentiability of Lipschitz functions on doubling metric measure spaces (v1). - ArXiv:1110.4279, 2011.

[Hei01] Heinonen, J.: Lectures on analysis on metric spaces. - Universitext, Springer-Verlag, New York, 2001.

[Hei07] Heinonen, J.: Nonsmooth calculus. - Bull. Amer. Math. Soc. (N.S.) 44:2, 2007, 163-232.

[HK98] Heinonen, J., and P. Koskela: Quasiconformal maps in metric spaces with controlled geometry. - Acta Math. 181:1, 1998, 1-61.

[Kei04a] KеIтн, S.: A differentiable structure for metric measure spaces. - Adv. Math. 183:2, 2004, $271-315$.

[Kei04b] KeIth, S.: Measurable differentiable structures and the Poincaré inequality. - Indiana Univ. Math. J. 53:4, 2004, 1127-1150.

[KM11] Kleiner, B., and J. MaCKAY: Differentiable structures on metric measure spaces: A Primer. - ArXiv:1108.1324, 2011.

[dL62] DE Leeuw, K.: Banach spaces of Lipschitz functions. - Studia Math. 21, 1961/1962, $55-66$.

[Wea99] Weaver, N.: Lipschitz algebras. - World Sci. Publ., River Edge, NJ, 1999.

[Wea00] Weaver, N:: Lipschitz algebras and derivations. II. Exterior differentiation. - J. Funct. Anal. 178:1, 2000, 64-112. 